\title{
INNOVATIVE ANWENDUNGEN MIT MOBILITÄTSDATEN: INTERNATIONALE FALLBEISPIELE
}

Eine Studie der mFUND-Begleitforschung des WIK

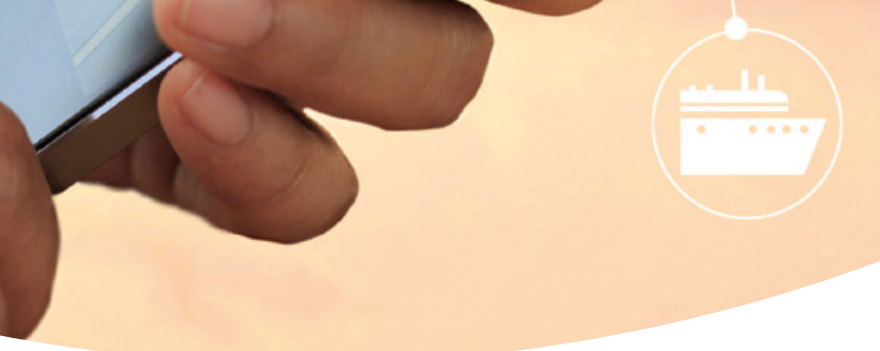

Autoren:

Alex Dieke

Dr. Sebastian Tenbrock Sonja Thiele Julia Wielgosch

Oktober 2018 


\section{Impressum}

WIK Wissenschaftliches Institut für

Infrastruktur und Kommunikationsdienste $\mathrm{GmbH}$

Rhöndorfer Str. 68

53604 Bad Honnef

Deutschland

Tel.: $\quad+4922249225^{-0}$

Fax: $\quad+4922249225-63$

eMail: info(at)wik.org

www.wik.org

\section{Vertretungs- und zeichnungsberechtigte Personen}

Geschäftsführer und Direktor

Dr. Iris Henseler-Unger

Direktor

Abteilungsleiter

Post und Logistik

Alex Kalevi Dieke

Direktor

Abteilungsleiter

Netze und Kosten

Dr. Thomas Plückebaum

Leiter Verwaltung

Karl-Hubert Strüver

Vorsitzende des Aufsichtsrates

Dr. Daniela Brönstrup

Handelsregister

Steuer Nr.

Amtsgericht Siegburg, HRB 7225

222/5751/0722

Umsatzsteueridentifikations Nr. DE 123383795 
INHALTSVERZEICHNIS

ZUSAMMENFASSUNG $\quad 2$

$\begin{array}{ll}\text { SUMMARY } & 2\end{array}$

1 WIRTSCHAFTLICHE POTENZIALE INNOVATIVER DATENANWENDUNGEN 3

2 FALLBEISPIEL NORDICWAY 6

3 FALLBEISPIEL SMART PORT ESTLAND 11

4 FALLBEISPIEL TRAFI $\quad 15$

5 FALLBEISPIEL SMART 19

6 FALLBEISPIEL SAFEMOTOS

7 FALLBEISPIEL URBANCYCLERS

8 SCHLUSSFOLGERUNGEN UND ERKENNTNISSE FÜR DEUTSCHLAND 31 


\section{ZUSAMMENFASSUNG}

Die Digitalisierung hat das Potenzial, den Markt für Mobilität zukünftig radikal zu beeinflussen. Bereits heute haben digitale datenbasierte Anwendungen den Verkehrssektor verändert. Navigationshelfer, Planungshelfer für den ÖPNV und Wettermelder sind aus privatem Alltag und geschäftlichem Einsatz nicht mehr wegzudenken. Die zunehmende Digitalisierung und Vernetzung der Verkehrsinfrastruktur und neue Technologien ermöglichen es, Daten verschiedener Quellen miteinander zu verknüpfen und somit große Datenmengen zu generieren und auszuwerten. Komplexe Analysemodelle ermöglichen dabei neue Erkenntnisse und Analyseergebnisse in „Echtzeit“. Innovative Anwendungen haben das Potenzial, den Verkehr verlässlicher, sicherer, kostengünstiger und umweltfreundlicher zu organisieren und Wachstumsimpulse für die Wirtschaft zu generieren.

Diese Studie der mFUND-Begleitforschung des WIK stellt sechs Fallbeispiele für erfolgreiche, innovative Anwendungen im Ausland vor. Sie geben Einblick in mögliche Einsatzfelder und Umsetzungsstrategien datenbasierter Innovationen in den Bereichen Verkehrssicherheit, Hafenmanagement, Verkehrsplanung, intermodale Routenplanung und nachhaltige Mobilität und können mFUND-Projekten und anderen Vorhaben, die innovative Nutzungskonzepte erforschen und entwickeln, wertvolle Anregungen liefern. Die Schlussfolgerungen stellen besonders interessante Aspekte der einzelnen Anwendungen für Deutschland heraus.

\section{SUMMARY}

Digitisation opens potentials to affect radical changes for mobility markets. Digital, data-based applications are changing the transport and mobility sector today and will continue to bring about innovation and efficiency improvements. Navigation tools for private and public transport and weather applications have become ubiquitous for many private and business users. Advances in digitisation, connected transport infrastructures and new technology offer ever-growing opportunities to combine and enrich data from different sources, and generate and process huge amounts of data. Complex models and analytical tools offer new potentials to organise transport more reliably, more securely, more cost-effectively, and more sustainably. At the same time, they promote economic growth and efficiency.

This study was prepared by the accompanying research for mFUND, a financial assistance programme sponsored by the German Federal Ministry of Transport and Digital Infrastructure to promote digital innovations for mobility in Germany. This study presents six international case studies for successful, innovative applications. They illustrate the diverse use cases and strategies of data-based innovation in transport security, management of seaports, traffic planning, intermodal routing and sustainable mobility. The case studies offer insights and learnings for projects sponsored by mFUND that research and develop innovative technologies and applications in Germany. 


\section{WIRTSCHAFTLICHE POTENZIALE INNOVATIVER DATENANWENDUNGEN}

Die Digitalisierung hat das Potenzial, den Markt für Mobilität zukünftig radikal zu beeinflussen. Bereits heute haben digitale datenbasierte Anwendungen den Verkehrssektor verändert. Navigationshelfer, Planungshelfer für den ÖPNV und Wettermelder sind aus privatem Alltag und geschäftlichem Einsatz nicht mehr wegzudenken. Die zunehmende Digitalisierung und Vernetzung der Verkehrsinfrastruktur wandelt das Verständnis und die Nutzungsmöglichkeiten von Fahrzeugen und verändert tradierte Geschäftssysteme. Neue Technologien und Analysemodelle ermöglichen es, Daten verschiedener Quellen miteinander zu verknüpfen und die stetig zunehmenden Datenmengen auszuwerten. Diese stammen unter anderem aus den Sensoren der Fahrzeuge und der Verkehrsinfrastruktur oder sie werden durch die Verkehrsteilnehmer selbst erzeugt und zur Verfügung gestellt. Komplexe Analysemodelle ermöglichen dabei neue Erkenntnisse und Analyseergebnisse in „Echtzeit".

Die Studie analysiert internationale Fallbeispiele für erfolgreiche, innovative Anwendungen. Befördert durch neue technologische Möglichkeiten entstehen weltweit digitale Innovationen als Antwort auf Herausforderungen wie steigende Verkehrsaufkommen, Lärm- und Luftverschmutzung. Die vorgestellten Best Practices geben Einblick in mögliche Anwendungsbereiche und Umsetzungsstrategien für datenbasierte Innovationen im Mobilitätssektor und können mFUND-Projekten und anderen Vorhaben, die innovative Nutzungskonzepte erforschen und entwickeln, wertvolle Anregungen liefern. Für die Studie wurden sechs internationale Anwendungen ausgewählt, die mit Mobilitäts-, Geo- oder Wetterdaten arbeiten. Sie stellen Beispiele für neu entwickelte Produkte oder innovative Erweiterungen bereits bekannter Lösungen dar. Die Auswahl soll einerseits das breite Spektrum der möglichen Anwendungen verdeutlichen und andererseits Anregungen aus verschiedenen Ländern für den deutschen Markt bieten.

Digitale datenbasierte Anwendungen bieten die Chance, den Verkehr verlässlicher, sicherer, kostengünstiger und umweltfreundlicher zu organisieren. Sie haben das Potenzial, die optimale Streckenwahl, Geschwindigkeit und Abstand der Verkehrsmittel sowie die effiziente Planung und Nutzung von Infrastrukturen zu unterstützen - für den Personenverkehr und die Logistik.

Verkehrsteilnehmer können mit Hilfe besserer Mobilitätsangebote und einer bedarfsgerechteren Routenplanung erhebliche Zeit- und Kostenersparnisse realisieren. Intelligente Navigationssysteme berücksichtigen aktuelle Informationen, z. B. zur Verkehrsauslastung und Wetterlage, und ermöglichen eine dynamische Anpassung der Streckenplanung. Neue Mobilitätsdienstleistungen wie Sharing-Dienste erweitern das Mobilitätsangebot und beflügeln den Wettbewerb auf dem Transportmarkt, was geringere Preise und bessere Services für die Verbraucher erwarten lässt.'

Städte können von einem effizienteren Verkehrssystem profitieren. Verkehrslärm, Flächenknappheit und Feinstaubbelastungen sind nur einige der aktuellen Herausforderungen, mit denen sich Städte konfrontiert sehen und für die digitale Innovationen Antworten liefern. Indem große Datenmengen zusammengeführt und gemeinsam ausgewertet werden, können Verkehrsunternehmen ihr Angebot besser an den tatsächlichen Bedarfen ausrichten. Eine größere Kapazitätsauslastung und Flexibilisierung des Angebotes kann erreicht werden. Intermodale Routenplaner integrieren die verschiede- 
nen Angebote des ÖPNV mit Car- und Bikesharing-Diensten auf einer zentralen Plattform und ermöglichen so die nahtlose Verknüpfung von verschiedenen Verkehrsmitteln und -anbietern für eine Reise. Passgenau kann aus einem breiten Angebot ausgewählt werden, sodass individuelle Mobilität von Fahrzeugbesitz entkoppelt werden kann oder diesen ergänzt. ${ }^{2}$ Intelligente Navigationssysteme und digitale Parkplatzfinder erübrigen lange Suchfahrten und können ebenfalls dazu beitragen, das Verkehrsaufkommen in den Städten zu verringern. Für Kommunen und Verkehrsplaner stellen Daten aus Verkehrssicherheitsscreenings zudem wichtige Entscheidungsgrundlagen für eine effiziente und sichere Infrastrukturplanung dar. Verkehrswege können automatisch auf ihre Belastung überwacht werden, um Instandhaltungsmaßnahmen effizient zu planen. Darüber hinaus können Anwendungen auch genutzt werden, um den Verkehr zu lenken, Engstellen zu entlasten und die Nutzung alternativer Transportmittel zu fördern.

Auch der ländliche Raum kann von diesen digitalen Mobilitätslösungen profitieren. Schwach besiedelte Regionen stehen vor der Herausforderung, ein verlässliches Mobilitätsangebot bei geringer Nachfrage sicherzustellen. On-Demand Shuttledienste ohne feste Routen und Fahrpläne können den traditionellen ÖPNV ergänzen. ${ }^{3}$ Digitale Plattformen vergrößern und bündeln das Mobilitätsangebot und liefern aktuelle Informationen über Veränderungen und Neuigkeiten.

Innovative datenbasierte Anwendungen bieten darüber hinaus eine große Chance für eine klimaund umweltverträglichere Mobilität. Der Verkehr ist der drittgrößte Verursacher von Emissionen in Deutschland, die Emissionen resultieren dabei zum Großteil aus dem Straßenverkehr. ${ }^{4}$ Viele Städte kämpfen heute mit hohen Schadstoff- und Lärmbelastungen, die die Gesundheit beeinträchtigen können. ${ }^{5}$ Datenbasierte Innovationen können dazu beitragen, die mobilitätsbedingten Emissionen zu verringern, indem sie den Verkehr effizienter gestalten und die Attraktivität umweltfreundlicher Verkehrsmittel erhöhen.

Die Analyse von Unfallstatistiken und damit zusammenhängenden Daten können zudem einen Beitrag zu mehr Verkehrssicherheit leisten. Innovative Anwendungen können neue Erkenntnisse über Gefahrenstellen liefern und diese passgenau an die Verkehrsteilnehmer kommunizieren. Rettungsfahrzeuge können von einer auf ihren Einsatz abgestimmten Verkehrsführung profitieren und so schneller an ihren Einsatzort gelangen.

Umfassende Verkehrsinformationen und intelligente Verkehrsnetze sind wichtige Wettbewerbsfaktoren für die Logistikbranche. ${ }^{6}$ Anwendungen, die die großen Datenmengen aus der Logistikinfrastruktur erschließen und deren Analyse in Echtzeit ermöglichen, erlauben eine effiziente Planung und Steuerung der Warenströme. Routen können bedarfsgerecht geplant und beispielsweise auf die besonderen Erfordernisse von Schwerlasttransporten abgestimmt werden.

Mobilitäts-, Geo- und Wetterdaten können auch für Innovationen außerhalb des Verkehrssektors genutzt werden. Daten aus Fahrzeugsensoren oder aus Sensornetzen können beispielsweise bisherige Wetter- und Umweltdaten ergänzen und dazu beitragen, Vorhersagemodelle zu verbessern.

Vgl. Goodall, Fishman, Bornstein, Bonthron (2017): The rise of mobility as a service, in: Deloitte Review, Issue 20, S.113-129. Vgl. „On-Demand“ ÖPNV als Chance für ländlichen Raum?, URL: www.dstgb.de/dstgb/Homepage/Aktuelles/Archiv/Archiv 2017/ "On-Demand" ÖPNV als Chance für ländlichen Raum\%3F/. BMUB (2017): Klimaschutz in Zahlen, S. 37.

Vgl. Kessinger (2017): Luftqualität 2016 in Deutschland, in: UMID, Nr.01/2017, S.5-11; Wothge (2016): Die körperlichen und psychischen Wirkungen von Lärm, in: UMID, Nr.01/2016, S.38-43.

Fraunhofer-Institut für System- und Innovationsforschung (2012): Gesamtwirtschaftliche Potenziale intelligenter Netze in Deutschland, S. 30-31. 
Neben Effizienzsteigerungen bieten innovative digitale Anwendungen ein großes Potenzial, neue Wachstumsimpulse zu generieren. Durch die innovative Kombination und Nutzung von Daten können neue Geschäftsmodelle mit großem Wachstumspotenzial entstehen.7 Eine Studie des Fraunhofer Institutes beziffert das Einsparpotenzial intelligenter Mobilitäts- und Logistikkonzepte mit insgesamt 8 Mrd. Euro jährlich. Zusätzlich seien Wachstumsimpulse in Höhe von ca. 2 Mrd. Euro jährlich möglich. ${ }^{8}$ Laut des Monitoring-Reportes Wirtschaft DIGITAL des Bundeswirtschaftsministeriums trägt alleine die deutsche Informations- und Kommunikationstechnik-Branche knapp 105 Milliarden Euro zur gewerblichen Wertschöpfung in Deutschland bei. Mit deutschlandweit 93.00o Unternehmen und rund einer Million Beschäftigten ist sie ein wichtiger Beschäftigungsfaktor in Deutschland. ${ }^{9}$ 


\title{
2 FALLBEISPIEL NORDICWAY
}

\author{
Dänemark, Finnland, Norwegen, Schweden
}

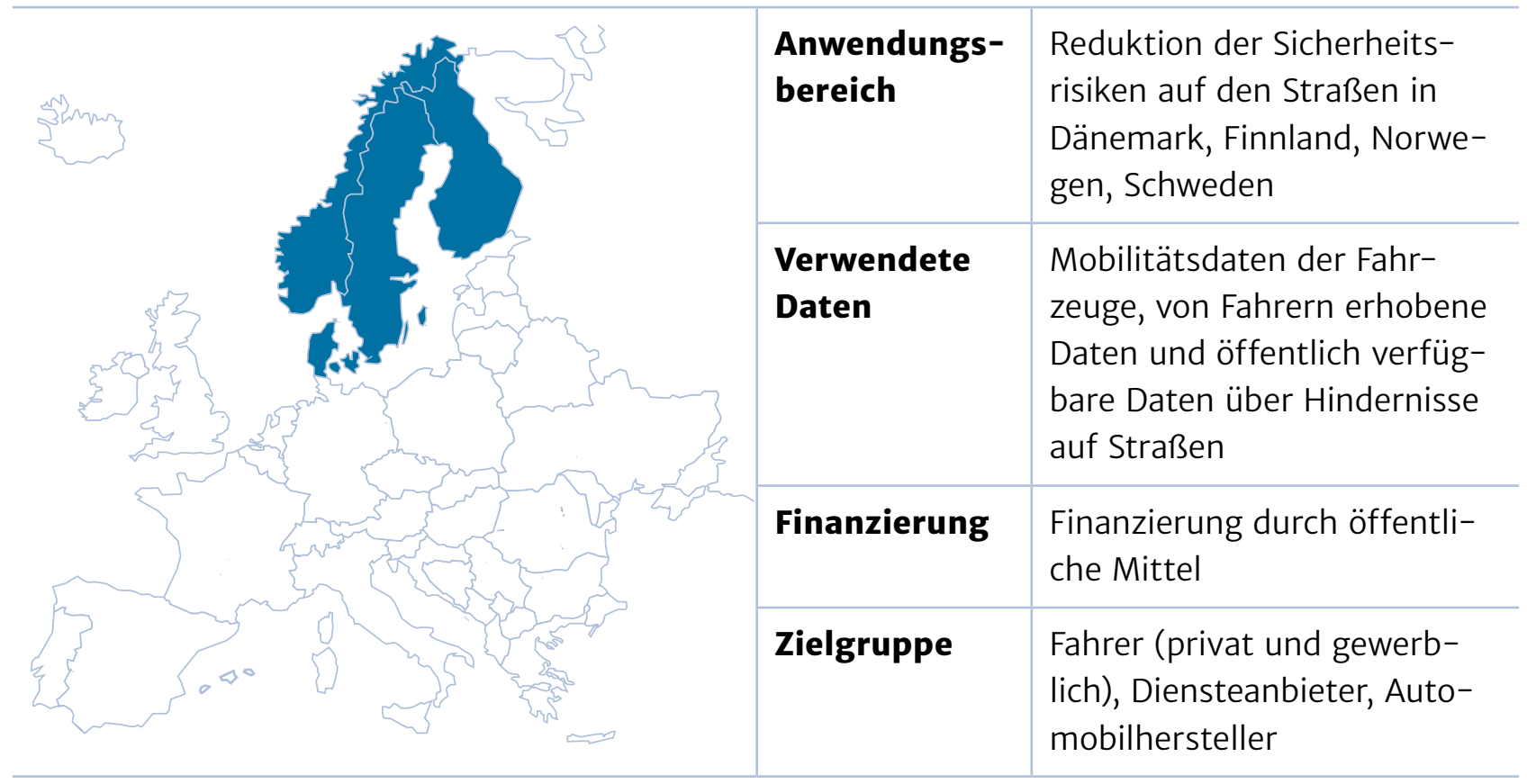

\section{BESCHREIBUNG DER ANWENDUNG}

NordicWay ist ein länderübergreifendes Projekt zur Erhöhung der Verkehrssicherheit, an dem Dänemark, Finnland, Norwegen und Schweden beteiligt sind. In einem Straßennetzwerk in den skandinavischen Ländern werden dabei standortbezogene Sicherheitsrisiken an die Fahrer und öffentliche Behörden kommuniziert: Die Nutzer von NordicWay erhalten Informationen zu straßenbezogenen Sicherheitsrisiken mit sehr geringer zeitlicher Verzögerung, bspw. zu Hindernissen auf der Fahrbahn, Unfällen, Glätte, StraBenarbeiten und Witterungsbedingungen.

Ziel von NordicWay ist es, schneller und umfassender auf potenzielle Gefahren und Notfälle reagieren zu können und dadurch die Verkehrssicherheit zu erhöhen. Da ein Großteil der Vor- und Unfälle zu Verkehrsstaus führt, soll auch der Verkehrsfluss verbessert, die Emissionen reduziert und die Effizienz gesteigert werden. Bis 2030 soll durch eine weitreichende Nutzung von NordicWay die Zahl der Unfälle mit Verletzungen und Todesfolge in dem betroffenen Straßennetzwerk deutlich reduziert werden.

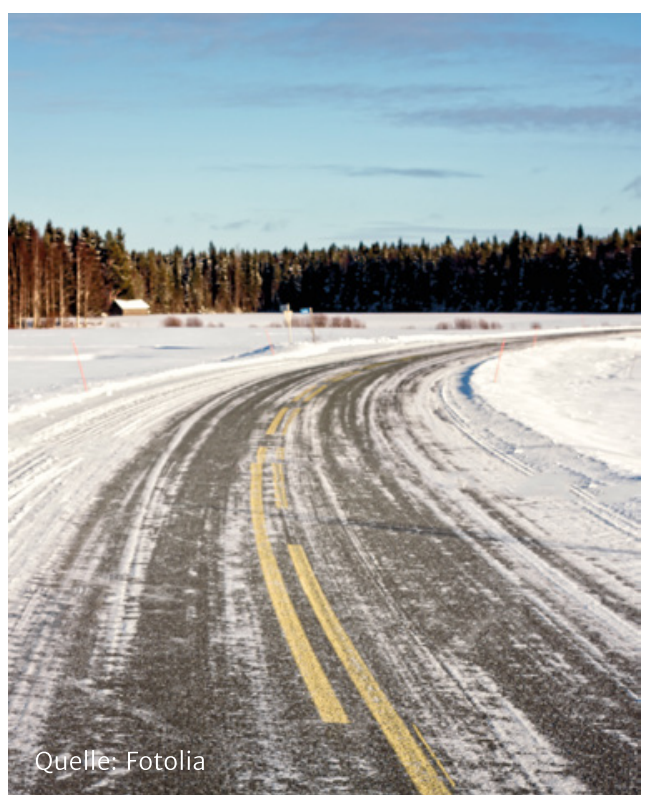

Abbildung 1: Fahrer erhalten Informationen zu Hindernissen auf der Fahrbahn, Unfällen und Witterungsbedingungen 
Die Besonderheit von NordicWay besteht darin, dass das Netzwerk eine vollständige Straßenabdeckung in den betroffenen Gebieten gewährleistet. Das geografische Gebiet von NordicWay zeichnet sich durch ein sehr langes Straßennetz von meist überregionalen Verkehrswegen aus; in Teilen herrscht dort ein verhältnismäßig geringes Verkehrsaufkommen. Gleichzeitig gibt es dort aber auch häufig Schnee und Glatteis. ${ }^{10}$

Das Projekt ist als Kooperation zwischen öffentlichen und privaten Partnern aufgesetzt. Die Zusammensetzung wird in der folgenden Tabelle 1 dargestellt:

\begin{tabular}{l|l|lll} 
& Dänemark & Finnland & Norwegen & Schweden \\
\hline $\begin{array}{l}\text { Öffentliche } \\
\text { Partner }\end{array}$ & $\begin{array}{l}\text { Danish Road } \\
\text { Directorate }\end{array}$ & $\begin{array}{l}\text { Finnish Transport } \\
\text { Agency }\end{array}$ & $\begin{array}{l}\text { Norwegian Public } \\
\text { Road Administ- } \\
\text { ration }\end{array}$ & $\begin{array}{l}\text { Swedish Trans- } \\
\text { port Administ- } \\
\text { ration }\end{array}$ \\
\hline Private Partner & & HERE, & Volvo Cars & Ericsson Kapsch \\
& & Infotripla & & $\begin{array}{l}\text { Traffic Com } \\
\text { Scania Volvo Cars }\end{array}$
\end{tabular}

Tabelle 1: Öffentliche und private Partner bei NordicWay"1

Bereits seit 1995 gibt es Kooperationen zwischen den vier skandinavischen Ländern, um die Effizienz und die Sicherheit ihrer Verkehrssysteme zu steigern.

\section{TECHNISCHE UMSETZUNG}

Die technische Umsetzung der Kommunikation stellt die Grundlage für den Erfolg von NordicWay dar. Im Projekt muss eine sichere und zuverlässige Kommunikation über kurze, mittlere und lange Entfernungen sichergestellt werden. Informationen werden dabei zwischen den Fahrzeugen, der Verkehrsinfrastruktur und den Projektbeteiligten ausgetauscht. Bei der Umsetzung des Projektes strebten die Projektbeteiligten an, möglichst große Teile der bestehenden Infrastruktur - sowohl der Kommunikationsverbindungen als auch der physischen Infrastruktur - nutzen zu können. Eine schematische Darstellung der Kommunikationsarchitektur findet sich in Abbildung 2. 


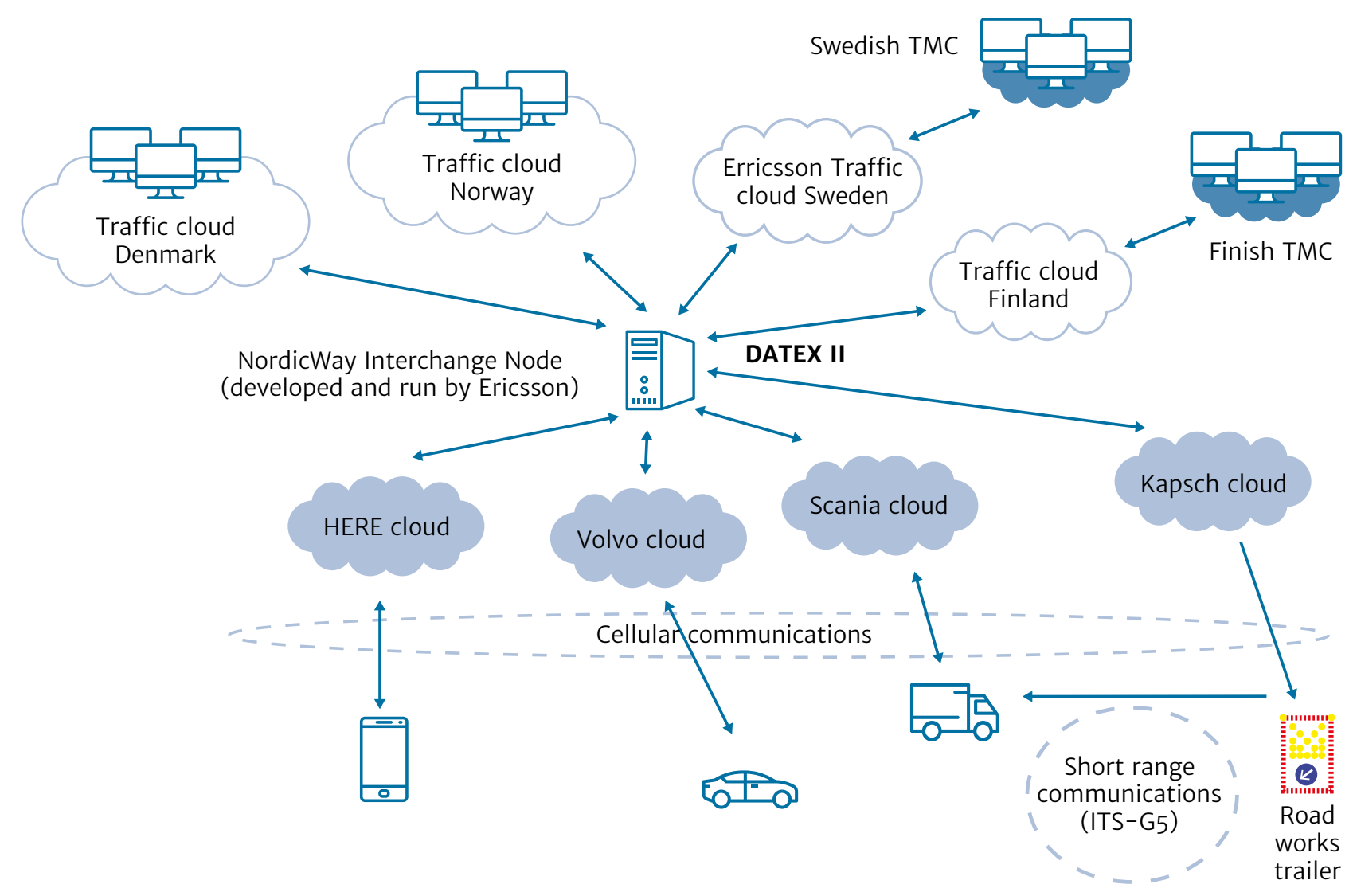

Abbildung 2: Kommunikationsarchitektur von NordicWay ${ }^{12}$

Die Kommunikation über kurze Entfernungen erfolgt auf der Grundlage von WLAN-basierter Direktkommunikation. Hierfür wurden eigens Standards, Prozesse, Schnittstellen und Sicherheitsvorkehrungen entwickelt. Die bislang nicht vorhandene Kommunikation über mittlere und lange Entfernungen wurde durch NordicWay auf Basis von Mobilfunktechnologien ergänzt, da in dem Gebiet eine sehr gute Abdeckung durch Mobilfunkkonnektivität (3G und LTE) vorliegt. Bei der Implementierung der Kommunikation über mittlere und lange Entfernungen wurde ein besonderer Fokus auf grenzüberschreitende Interoperabilität und Standards gelegt, so dass physische Grenzübergänge den Informationstransfer nicht beeinträchtigen.

Insofern entsteht durch NordicWay nun erstmalig eine auf Mobilfunk und C-ITS (Cooperative Intelligent Transport Systems) basierende Kommunikationslösung in großräumigem Umfang; in diesem Zusammenhang wird auch von einer Konvergenz der Kommunikationslösungen oder hybrider Kommunikation gesprochen. ${ }^{13}$ 


\section{DATENBASIS UND -VERWENDUNG}

NordicWay stellt den Nutzern Informationen und Dienste grenzüberschreitend auf Basis des oben beschriebenen Kommunikationsnetzes bereit. ${ }^{14}$ Die Informationen setzen sich aus zwei Quellen zusammen: einerseits von den Nutzern selbst erhobene Daten und andererseits Daten, die von öffentlichen Akteuren und privaten Unternehmen (Fahrzeugflotten, Navigationssystemen) bereitgestellt werden. So sammelten in der ersten Projektphase (bis Ende 2017) mehrere tausend Fahrer Daten auf Grundlage der Sensoren in den Fahrzeugen. Diese wurden in Verbindung mit den externen Datenquellen in Cloud-Systemen gespeichert und ausgewertet. Im Anschluss wurden (geospezifisch) relevante Informationen den Fahrern und der Verkehrsinfrastruktur zur Verfügung gestellt. Fahrer können diese etwa über Apps oder den Bordcomputer ihres Fahrzeugs abrufen. ${ }^{15}$

Die zweite Projektphase startete zu Beginn des Jahres 2018: Neben einer höheren Zahl von Nutzern und weiteren Strecken sollen neue Anwendungen getestet werden, beispielsweise im Bereich des automatisierten Fahrens. Hierfür wurden eigens Straßenabschnitte ausgewiesen. Für Unternehmen und die beteiligten Stakeholder soll sich NordicWay als datenbasiertes Ökosystem stets weiterentwickeln; auf Grundlage der gesammelten und ausgewerteten Daten sollen kontinuierlich innovative Dienste konzipiert und angeboten werden. ${ }^{16}$

\section{FINANZIERUNG}

Die Finanzierung vom NordicWay erfolgt durch öffentliche Mittel der jeweiligen Länder. Für die Nutzer fallen keine Kosten an.

Das Projekt wird von der Europäischen Union im Rahmen des Programms „Connecting Europe Facility" bis zu 50\% (in den Ländern verschieden) kofinanziert.

\section{ERFOLG}

Nach der ersten Projektphase wurden private und gewerbliche Nutzer von NordicWay in Finnland zu ihrer Einschätzung im Hinblick auf die bereitgestellten Informationen befragt. Hier zeigt sich ein positives Bild, auch wenn eine relativ geringe Zahl von Nutzern an der Befragung teilgenommen hat. Etwa $57 \%$ der Nutzer gaben an, dass durch NordicWay mögliche Risiken präziser und schneller angezeigt werden. Zudem teilten ca. $45 \%$ der Nutzer mit, dass ihnen die Störungen nicht durch andere Informationsquellen mitgeteilt werden. Immerhin 95\% der erfahrenen und etwa $86 \%$ der weniger erfahrenen Nutzer gaben an, dass sie die App weiterhin nutzen wollen (vor allem auf Basis ihres Smartphones). Nur etwa 11\% der Nutzer teilten mit, dass ihnen durch die App kein zusätzlicher Nut-

\footnotetext{
$14 \quad$ Landesspezifisch können über NordicWay noch weitere Dienste bereitgestellt werden.

15 Vgl. NordicWay (2017): NordicWay - final report, URL: www.vejdirektoratet.dk/EN/roadsector/Nordicway/Documents/NordicWay_Final\%20Report.pdf.

16 Vgl. NordicWay (2017): Deployment Roadmap - Recommendations for future work, URL: www.vejdirektoratet.dk/EN/roadsector/ Nordicway/Documents/NordicWay\%20Roadmap\%20M_12\%20(secured).pdf.
} 
zen entsteht.

Die größten Vorteile sehen die Nutzer bei der Anzeige von Hindernissen auf der Straße; hier sagten etwa $80 \%$ der Nutzer aus, dass diese immer oder in den meisten Fällen angezeigt werden. Nach den Warnungstypen der App befragt, priorisierten die Nutzer straßenbezogene Warnungen - wie Unfälle, Hindernisse, Glätte und Straßenarbeiten - als wichtiger gegenüber wetterbezogenen Warnungen - wie außergewöhnliche Witterung und schlechte Sicht. Zudem gaben sie an, dass sie sich zusätzlich Warnungstypen wünschen, etwa zu Verkehrsstauungen, (größeren) Tieren auf den Straßen (Elche und Rentiere), anormalem Fahrerverhalten sowie Polizei- und Verkehrskontrollen. ${ }^{17}$

\section{PERSPEKTIVE}

In der zweiten Projektphase werden noch mehr Nutzer über Endgeräte und den Bordcomputer der Fahrzeuge eingebunden und das Straßennetzwerk wird ausgeweitet. Bei der Umsetzung des Projektes wurde großer Wert auf Skalierbarkeit gelegt, so dass zukünftig mehr Nutzer und neue Nutzergruppen berücksichtigt werden können. Beispielsweise könnten Konzepte im Bereich „Mobility as a Services" (MaaS) getestet werden. ${ }^{18}$ Zudem werden momentan cloudbasiert Anwendungsfelder im Bereich des automatisierten und autonomen Fahrens auf Grundlage von NordicWay entwickelt und getestet. Perspektivisch soll NordicWay auch auf andere europäische Ländern ausgeweitet werden. ${ }^{19}$

\section{Besonders interessant für Deutschland:}

- Öffentliche Akteure können gerade bei internationalen Projekten maßgeblicher Initiator sein und länderübergreifende Problemstellungen aufgreifen.

- Ein zentrales Ziel der Anwendung, die technische Übermittlung von Verkehrsstörungen über sehr lange Distanzen, erscheint auch für Deutschland als Transitland sehr relevant.

- Die Verbreitung einer Anwendung kann dadurch erhöht werden, dass sie von den Endkunden über verschiedene Kanäle genutzt werden kann (etwa via Smartphone und Bordcomputer im Auto).

- Die Verwendung von offenen Schnittstellen zwischen den Projektpartnern erleichtert die Aufnahme von weiteren Partnern zu einem späteren Zeitpunkt.

17 Vgl. NordicWay (2017): Evaluation Outcome Report - Finland, URL: www.vejdirektoratet.dk/EN/roadsector/Nordicway/Documents/NordicWay\%20evaluation\%20outcome\%2onational\%20report_Finland_Final.pdf.

18 Mobility as a Services umschreibt die Planung (und Abrechnung) eines Reisewegs von privaten und öffentlichen Verkehrsmitteln auf der Plattform. Bedarfsspezifisch kann auf dieser Plattform etwa der günstige oder schnellste Verkehrsweg aus einer Kombination von vorgegebenen Verkehrsmitteln zusammengestellt werden.

19 Vgl. NordicWay (2017): Deployment Roadmap - Recommendations for future work, URL: www.vejdirektoratet.dk/EN/roadsector/ Nordicway/Documents/NordicWay\%20Roadmap\%20M_12\%20(secured).pdf. 


\section{FALLBEISPIEL SMART PORT ESTLAND}

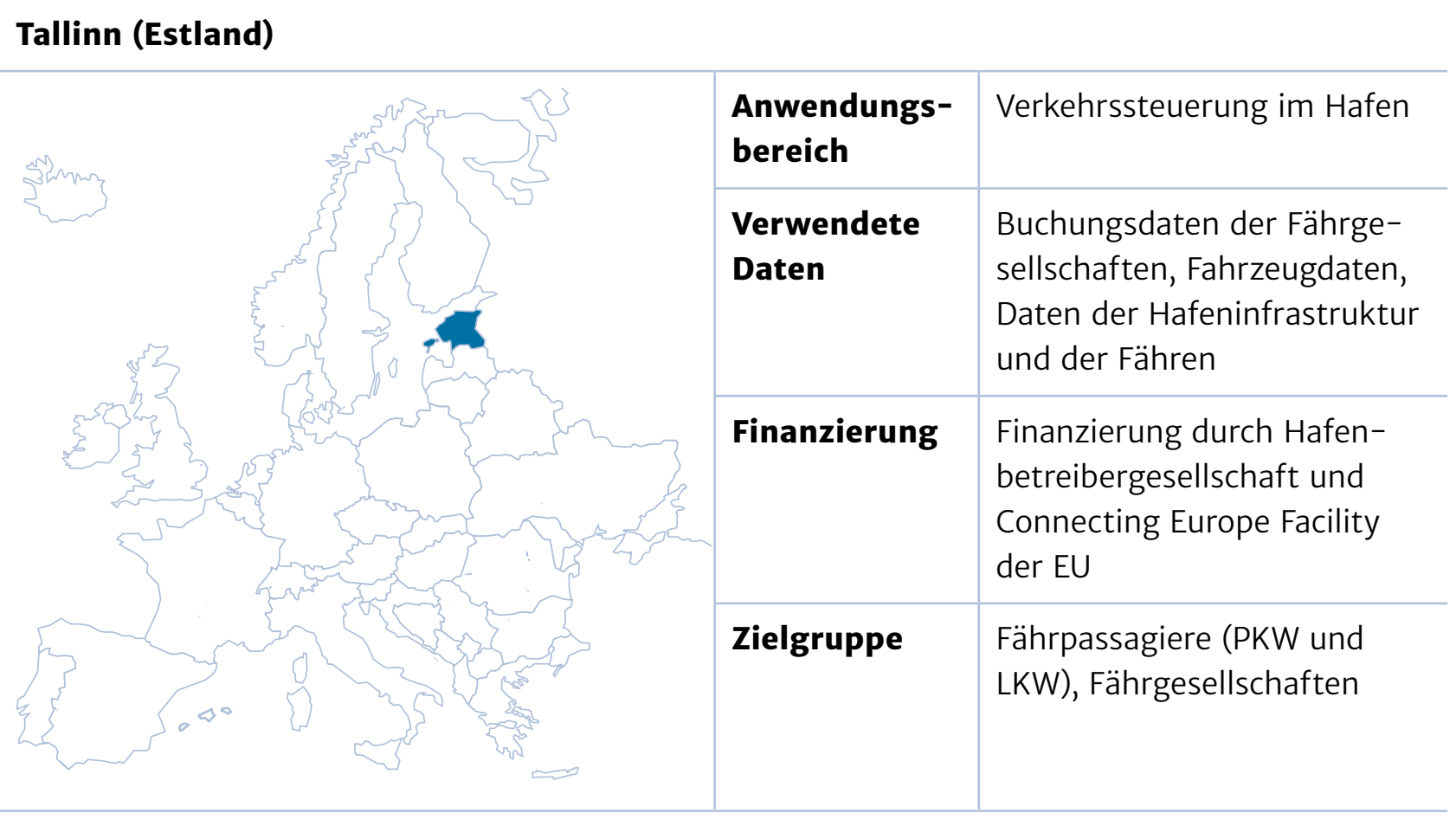

\section{BESCHREIBUNG DER ANWENDUNG}

Das Projekt Smart Port Estland hat das Ziel, die Wartezeiten für Autos und LKW an den Fähranlegern im Hafen der estnischen Hauptstadt Tallinn zu reduzieren. Um die wachsenden Verkehrsströme besser zu lenken, wurde ein innovatives Verkehrsmanagementsystem installiert.

Die Passagierzahlen sind in den letzten Jahren stark gestiegen, was den Hafen vor große Herausforderungen gestellt hat. Von 2012 bis 2015 hat sich die Zahl der Passagiere in Tallinn um ca. 10\% auf insgesamt fast 10 Millionen jährlich erhöht. ${ }^{20}$ Gleichzeitig ist die Fläche des Hafens begrenzt und kann nicht entsprechend erweitert werden. Für den Check-In der Passagiere stehen aufgrund der knappen Fläche nur wenige Kiosks zur Verfügung. Jede Reederei betrieb vor Start des Projektes eigene Schalter und nutzte zudem eigene Hard- und Softwarelösungen für den Abfertigungsvorgang. Personal eines externen Serviceanbieters wies den Fahrzeugen den Weg durch die Hafenanlage. Der langwierige und schwierig zu koordinierende Abfertigungsprozess führte zu langen Wartezeiten im Hafen. Passagiere mussten bis zu zwei Stunden vor Abfahrt am Hafen sein, um rechtzeitig an Bord zu gelangen. Unklare Verkehrs- und Richtungsanweisungen führten zudem dazu, dass Passagiere zum falschen Schiff geleitet wurden oder gefährliche Verkehrssituationen entstanden. Da die Passagiere ihre Fahrzeugmotoren während der langen Wartezeit laufen ließen, entstanden zudem hohe Emissionsbelastungen im Hafen. 


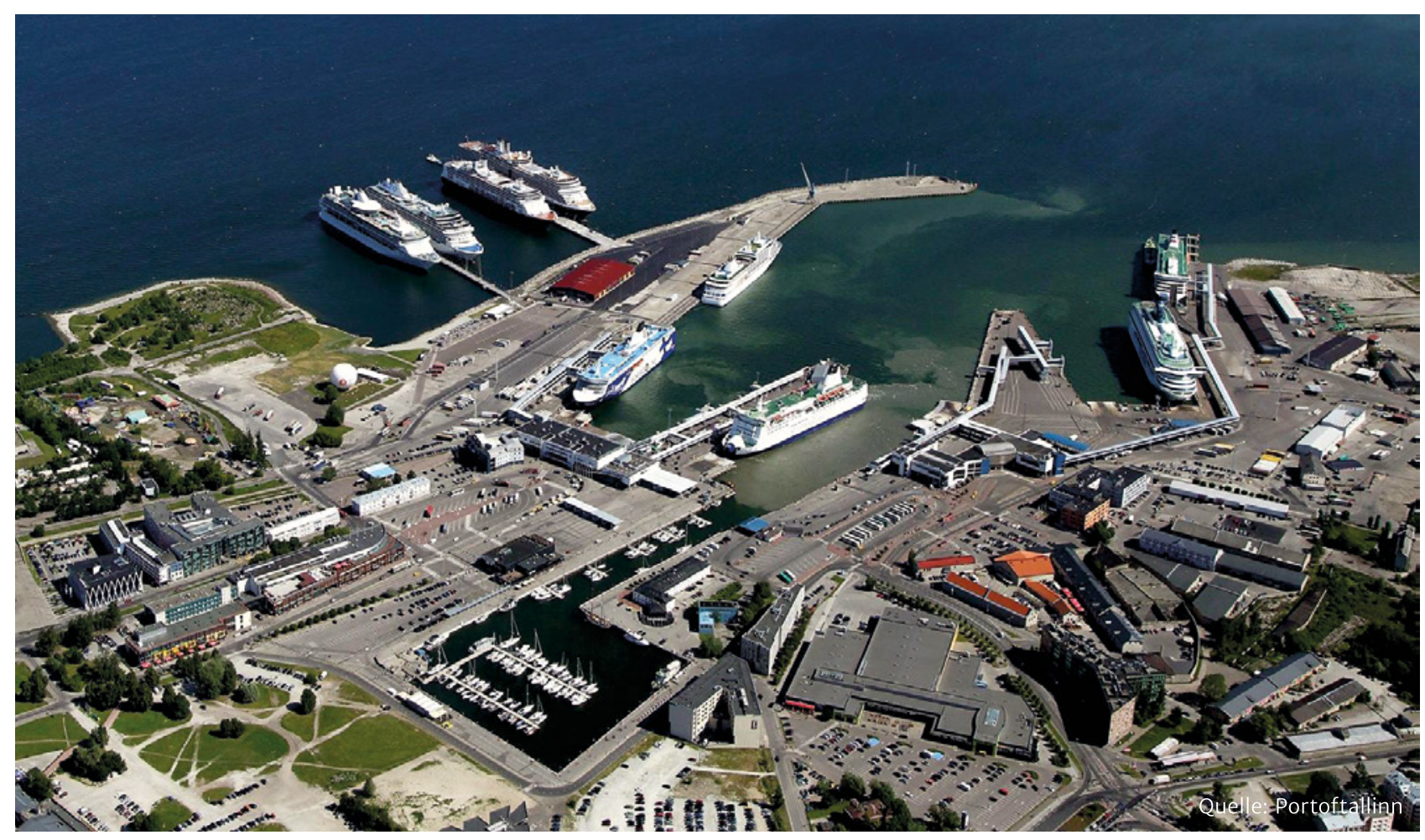

Abbildung 3: Wachsende Passagierzahlen bei gleichzeitig begrenzter Hafenfläche machten die Einführung eines neuen Verkehrsmanagementsystems erforderlich

Um den Abfertigungsvorgang schneller, einfacher und kundenfreundlicher zu gestalten wurde ein umfassendes, digitales Verkehrsmanagementsystem entwickelt. Die Fährbetreiber nutzen nun eine gemeinsame Plattform, auf der sie Daten ihrer Check-In-Systeme bereitstellen. Die Vorgänge wurden dabei weitestgehend automatisiert. Daten aller Fahrzeuge, die in den Hafen einfahren, werden erfasst und durch das System weiterverarbeitet. Unterstützt wird dies durch eine automatische Kennzeichenerkennung, die eine sichere und einfache Identifikation der Fahrzeuge ermöglicht. Das Hafensystem wertet die Fahrzeugdaten aus und steuert auf Grundlage dessen die die Verkehrszeichen und Schilder des digitalen Leitsystems sowie die automatischen Schranken, die zu den entsprechenden Wartezonen führen. Das automatische System gibt den Passagieren schnell und eindeutig Anweisungen, um die Schiffe bestmöglich beladen zu können.

Die Entwicklung des Projektes geschah in enger Zusam-

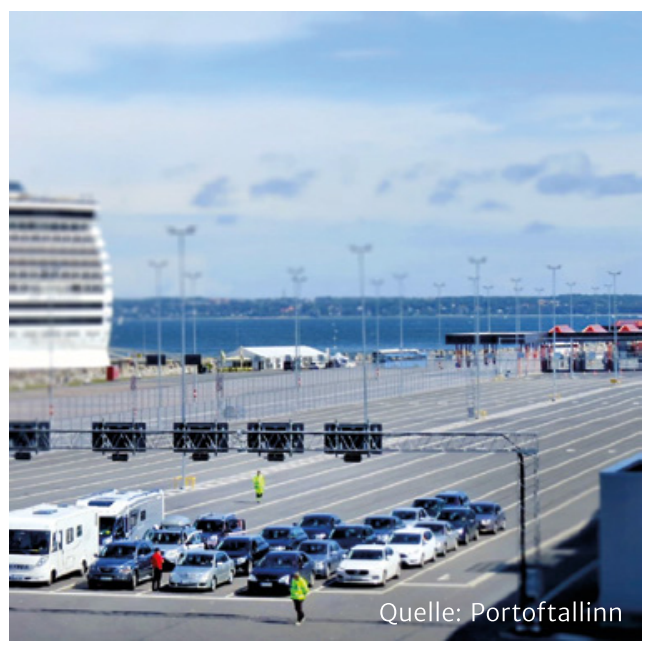

Abbildung 4: Das Hafensystem steuert die Verkehrszeichen und Schilder des digitalen Leitsystems sowie die automatischen Schranken, die zu den Wartezonen führen menarbeit mit den Fährbetreibern, die nach anfänglichem Zögern bald vom Potenzial des neuen Systems überzeugt waren. Verschiedene Häfen sind zudem als Partnerhäfen in das Projekt involviert. Die Häfen der Städte Stockholm (Schweden), Helsinki (Finnland) und Turku (Finnland) haben viele Fährverbindungen mit Tallinn. Um sicherzustellen, dass 
Prozesse für Fährbetreiber und Passagiere in diesen Häfen vergleichbar und kompatibel sind, haben sie an der Entwicklung von Smart Port Tallinn kooperiert. Kristiansand (Norwegen) ist ebenfalls als Partnerhafen involviert, da der Hafen sehr daran interessiert ist, die Innovationen des Smart Port Tallinn in Zukunft ebenfalls umzusetzen.

2014 begann die Entwicklung des Projektes. Seit Oktober 2017 werden die Lösungen des Smart Port Tallinn in einem ersten Terminal des Hafens eingesetzt, im Mai 2018 erfolgte die Implementierung in einem weiteren Terminal.

\section{DATENBASIS UND TECHNISCHE UMSETZUNG}

Sensoren erfassen beim Check-In automatisch Höhe, Breit und Länge sowie Gewicht (bei Lieferwagen) der Fahrzeuge. Diese Daten werden im zentralen Verkehrsmanagementsystem hinterlegt, mit den hinterlegten Buchungsdaten abgeglichen und dienen dazu, die Wagen bestmöglich auf die Fahrbahnen, die zu den Fähren führen, zu verteilen. Nach dem Check-In werden die Fahrzeuge mit Hilfe des digitalen Leitsystems in die entsprechenden Fahrbahnen eingewiesen. Ein Kamerasystem zur Nummernschilderkennung dient dazu, die Fahrzeuge jederzeit automatisch zu identifizieren und so sicherzustellen, dass sie sich am richtigen Ort befinden.

\section{FINANZIERUNG}

Smart Port Tallinn ist Teil des Twin-Port Projektes, ${ }^{21}$ das zum Ziel hat, den Güterverkehr zwischen den Häfen in Tallinn und in Helsinki zu harmonisieren: In beiden Häfen werden im Zuge des Projektes Investitionen in die Hafeninfrastruktur und -systeme durchgeführt, die den gegenseitigen Verkehrsfluss verbessern sollen. Das Projekt wird von der Europäischen Union im Rahmen des Programms „Connecting Europe Facility“ mit 20\% kofinanziert. Für den Hafen in Tallinn wurden im Rahmen des Projektes Investitionen in Höhe von insgesamt 13,3 Millionen Euro geplant. Die restlichen Mittel für Smart Port Tallinn werden von der Betreibergesellschaft des Hafens „Port of Tallinn“ gestellt.

\section{ERFOLG}

Der Abfertigungsprozess verläuft nach Projektumsetzung nun deutlich schneller und sicherer. Vor Projektstart mussten Passagiere mit PKWs etwa 45 bis 90 Minuten vor Abfahrt der Fähre am Hafen sein, um sich einzuchecken. Das neue System teilt den Passagieren Check-in-Zeiten zu, wodurch die Wartezeiten reduziert werden. Gleichzeitig sinkt der Ausstoß von Abgasen und CO2 im Hafen. Die Schiffe können deutlich schneller beladen und ihre Kapazitäten besser genutzt werden. Da das System größtenteils automatisch arbeitet, wird weniger Personal in den Kiosken und für die Arbeit als Einweiser benötigt, sodass ein wirtschaftlicher Betrieb sichergestellt werden kann. Da das System erst kürzlich vollständig implementiert wurde steht eine genaue Bewertung der Effizienzgewinne für den laufenden Betrieb noch aus. 


\section{PERSPEKTIVE}

Das im Rahmen des Projektes entwickelte System soll in Zukunft als fertiges Konzept für den internationalen Markt angeboten werden. Port of Tallinn plant, zusammen mit technischer Unterstützung vor Ort das System in interessierten Häfen zu installieren und in Zusammenarbeit mit den lokalen Akteuren anzupassen .

\section{Besonders interessant für Deutschland:}

- Die Einführung einer neuen, zentralen Lösung eröffnet die Möglichkeit, alte Strukturen aufzubrechen und Prozesse zielgerichtet neu zu gestalten.

- Eine Anwendung des Konzeptes auf andere Anwendungsfälle, wie bspw. das Parkflächenmanagement im Rahmen von Großveranstaltungen, ist denkbar.

- Eine effiziente Verkehrssteuerung erhöht die Kapazitäten und reduziert die Wartezeiten, ohne dass die Hafenfläche erweitert werden muss.

- Die Einbindung von Partnerhäfen stellt sicher, dass das erarbeitete Konzept auf andere Häfen übertragbar ist. 


\title{
4 FALLBEISPIEL TRAFI
}

\author{
Sao Paulo, Rio de Janeiro (Brasilien), Tallinn (Estland), Jakarta (Indonesien), Riga (Lett- \\ land), Vilnius, Kaunas, Klaipeda, Siauliai, Panevezys (Litauen), Madrid (Spanien), Taipei \\ (Taiwan), Istanbul, Ankara, Izmir, Bursa (Türkei)
}

\begin{tabular}{l|l}
\hline Zielgruppe & Verkehrsmittelübergreifender Routenplaner \\
\hline Ferwendete Daten & (Echtzeit-) Daten der Verkehrsanbieter, Nutzerdaten \\
\hline Finanzierung & Finanzierung durch Venture Capital-Geber \\
\hline Stadtbewohner und -besucher
\end{tabular}

\section{BESCHREIBUNG DER ANWENDUNG}

Trafi ist ein intermodaler Routenplaner. Auf Grundlage von Fahrplänen und Echtzeitinformationen der öffentlichen Verkehrsmittel, Verkehrslagedaten, Wetterdaten sowie Nutzerdaten ermöglicht die Anwendung eine genaue und verkehrsmittelübergreifende Reiseplanung in den Partnerstädten. Berücksichtigt werden verschiedene Verkehrsmittel, wie Busse und Bahnen des ÖPNV, Car- und Bikesharing-Angebote sowie Taxidienste. Ticketkauf und die Buchung eines Angebotes können komfortabel innerhalb der Anwendung durchgeführt werden. Trafi wird seit 2013 von einem litauischen Start-up entwickelt. Die Anwendung kann als Smartphone-App sowie als Webanwendung genutzt werden.

Trafi ermöglicht eine effizientere Fortbewegung in den Städten. Öffentliche Verkehrsmittel können komfortabel in die individuelle Routenplanung einbezogen werden und gewinnen gegenüber dem eigenen Auto an Attraktivität. Im Vergleich zu anderen am Markt befindlichen Routenplanern besticht 


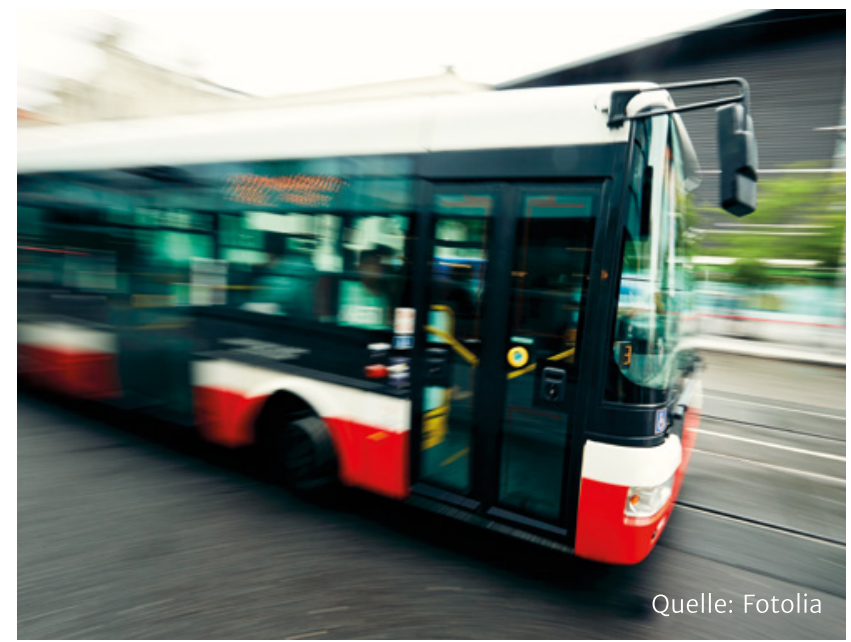

Abbildung 5: Trafi berücksichtigt Busse und Bahnen des ÖPNV, Car-Sharing, Bike-Sharing und Taxidienste
Trafi durch einen großen Funktionsumfang und eine sehr hohe Genauigkeit der Routenplanung, die erreicht wird indem Echtzeitverkehrsdaten und Nutzerdaten mit Hilfe von Algorithmen in Echtzeit ausgewertet werden. Trafi nutzt die Erkenntnisse aus der Datenauswertung, um die Routenplanung einerseits an die besonderen Gegebenheiten einer Stadt und andererseits an die individuellen Vorlieben der Nutzer anzupassen.

Die erhobenen und veredelten Daten können des Weiteren für eine bessere Verkehrsplanung der jeweiligen Städte genutzt werden. Trafi arbeitet unter anderem eng mit den Stadtverwaltungen von Vilnius und Jakarta zusammen. In Jakarta werden Algorithmen von Trafi genutzt, um an Bushaltestellen die Ankunftszeiten in Echtzeit anzuzeigen. Für die Verkehrsplanung von Vilnius stellt das Unternehmen Übersichten der städtischen Mobilitätsströme zusammen, die für eine bedarfsgerechtere und schnellere Infrastrukturplanung der Stadt genutzt werden.

\section{DATENBASIS UND -VERWENDUNG}

Trafi kombiniert für die Routenplanung Daten und Echtzeitinformationen verschiedener Quellen. Von Verkehrsbetrieben werden Soll-Fahrpläne, Haltestellen und Preisinformationen verwendet. Diese werden ergänzt durch GPS-Echtzeitdaten der Busse und Bahnen. Anbieter von Bike- und Car-Sharing sowie Taxidiensten stellen Informationen zu Stationen und Preisen sowie Echtzeitdaten zu Standorten und Verfügbarkeiten bereit. Des Weiteren gehen aktuelle und historische Verkehrslagedaten in die Routenplanung ein. Die Anwendung berücksichtigt für die Routenplanung zudem aktuelle Wetterdaten und Präferenzen der Nutzer für bspw. besonders preiswertes oder schnelles Reisen. Auf Grundlage von den in Vergangenheit gewählten Routen und Verkehrsmitteln sowie individueller Vorlieben werden individuelle Routenvorschläge ermittelt. Den Nutzern werden Informationen zu aktuellen Verkehrsereignissen in der App angezeigt. Diese werden einerseits von den Verkehrsunternehmen angegeben oder von den Nutzern selbst eingetragen und umfassen Hinweise zu aktuellen Baustellen, Staus, usw

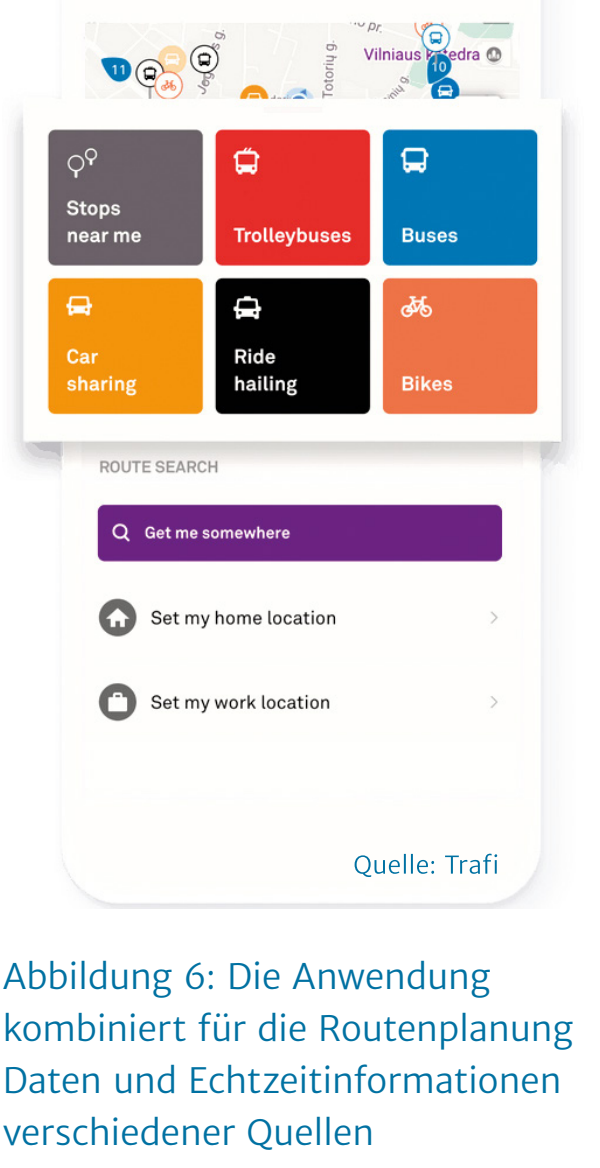




\section{FINANZIERUNG}

Die Entwicklung der Anwendung wird von Venture Capital-Gebern finanziert. In bislang vier Funding-Runden hat Trafi insgesamt $\$ 14,8$ Millionen von fünf Investoren erhalten. ${ }^{22}$ Trafis Geschäftsmodell basiert darauf, die Mobilitätsplattform sowie technische Lösungen an private Unternehmen zu lizensieren. Internationale Unternehmen wie Google, Apple und Lyft nutzen Lösungen von Trafi, um ihre Mobilitätsangebote anzubieten. Trafi bietet Verkehrsdaten aus seinem Datenpool für die Integration auf externen Webseiten, Apps und Anzeigetafeln an. Auch die Einbindung von Trafis Routendienst als Widget oder Button in Websites ist möglich. Die Anwendung selbst ist für die Nutzer kostenlos und beinhaltet keine Werbung.

\section{ERFOLG}

Trafi wird in bislang 16 Städten in 8 Ländern weltweit genutzt. In vielen Städten, wie in Vilnius und Jakarta, haben die Entwickler der Anwendung engen Kontakt mit den jeweiligen Stadtverwaltungen, die Trafi dabei unterstützen die relevanten Mobilitätsanbieter - allen voran der ÖPNV - auf der Plattform zu integrieren. Die App hat aktuell mehr als 4 Millionen Nutzer ${ }^{23}$ und eine durchschnittliche Nutzerbewertung von 4,3/5 Sternen auf Grundlage von über 60.000 Bewertungen im Google Playstore. 2016 wurde die App von der brasilianischen Stadt Rio de Janeiro zum besten Reiseplaner für die Olympischen Spiele gewählt. Die App wurde für die Wettkämpfe entsprechend angepasst und Informationen des Veranstaltungsplanes wurden integriert. Nach einer von Trafi selbst durchgeführten Umfrage wählen ca. 63\% der App-Nutzer in Istanbul häufiger öffentliche Verkehrsmittel, nachdem sie Trafi kennengelernt haben.

\section{PERSPEKTIVE}

Eine Expansion in weitere Großstädte und Länder, insbesondere auch in Deutschland, ist für die Zukunft geplant. Erfolgsentscheidend für die Etablierung als intermodaler Routenplaner in weiteren Städten ist insbesondere die Kooperation der öffentlichen Verkehrsanbieter mit Trafi und die Verfügbarkeit von (Echtzeit-) Daten der Verkehrsbetriebe. 


\section{Besonders interessant für Deutschland:}

- Anwendungen wie Trafi, die eine intermodale Routenplanung und Ticketkauf aus einer Hand ermöglichen, haben das Potenzial, die Verkehrsbelastung in Städten zu senken und den ÖPNV attraktiver zu machen.

- Für die Umsetzung des Konzeptes „Mobilität als Dienstleistung“ ist wesentlich, dass die wichtigsten Mobilitätsanbieter einer Stadt, insbesondere der ÖPNV, auf einer zentralen Plattform eingebunden werden.

- Für den Erfolg der Anwendung ist eine enge Kooperation zwischen den Entwicklern der Anwendung und den kommunalen Verkehrsbehörden wesentlich.

- Städte können die von der Anwendung gesammelten und aufbereiteten Daten für ein bessere Stadt- und Verkehrsplanung nutzen. 


\section{FALLBEISPIEL SMART}

\section{Enschede (Niederlande)}

\begin{tabular}{|l|l|l|}
\hline $\begin{array}{l}\text { Anwendungs- } \\
\text { bereich }\end{array}$ & $\begin{array}{l}\text { Verbesserung des innerstäd- } \\
\text { tischen Verkehrs in Enschede } \\
(\mathrm{NL})\end{array}$ \\
\hline $\begin{array}{l}\text { Verwendete } \\
\text { Daten }\end{array}$ & $\begin{array}{l}\text { Mobilitätsdaten der Nutzer, } \\
\text { Daten der Verkehrsbetriebe, } \\
\text { der kommunalen Verkehr- } \\
\text { überwachung und der Wet- } \\
\text { terdienste }\end{array}$ \\
\hline Finanzierung & $\begin{array}{l}\text { Entwicklung und Finanzie- } \\
\text { rung durch die Gemeinde } \\
\text { Enschede, Weiterentwicklung } \\
\text { u.a. durch das EU Projekt } \\
\text { EMPOWER }\end{array}$ \\
\hline Zielgruppe & $\begin{array}{l}\text { Kommunen, Stadtbewoh- } \\
\text { ner und -besucher, Pendler, } \\
\text { lokale Unternehmen }\end{array}$ \\
\hline
\end{tabular}

\section{BESCHREIBUNG DER ANWENDUNG}

SMART wurde von der Gemeinde Enschede initiiert, um die Verkehrssituation in der Stadt durch positive Anreize zu verbessern. Die Entwickler der Anwendung arbeiten in enger Abstimmung mit der Stadt zusammen, um mit Hilfe von SMART zur Umsetzung der kommunalen Verkehrsstrategie beizutragen. Die Nutzung umweltfreundlicher Verkehrsmittel oder die Wahl bestimmter, weniger befahrener Routen, wird mit Punkten belohnt („SMART Points"), die später im App-Shop für Gutscheine beteiligter lokaler Geschäfte eingelöst werden können.

Wandelt sich der Fokus der kommunalen Verkehrsstrategie, kann das Anreizsystem entsprechend angepasst werden. Lag der Schwerpunkt der Stadt Enschede zu Beginn der Arbeit mit SMART in der Entlastung eines bestimmten Verkehrsknotenpunktes, wird die Anwendung heute insbesondere genutzt, um den Fahrradverkehr in der Stadt zu fördern. Entsprechendes Verhalten wird mit Punkten honoriert. Die zu erlangenden Punkte richten sich auch nach den Vorsätzen der Nutzer. Sie können eintragen, ob sie künftig einen besonderen Wert auf umweltfreundliche Mobilität, mehr Bewegung oder besonders preiswertes Reisen legen wollen und bekommen höhere Punkte für entsprechendes Mobilitätsverhalten.

Auf Grundlage der kommunalen Verkehrsstrategie, der individuellen Vorsätze und des aufgezeichneten tatsächlichen Mobilitätsverhaltens der Nutzer werden personalisierte Herausforderungen erstellt. Nutzer können sich den Herausforderungen alleine stellen oder sich über die App mit anderen 
Nutzern zum gemeinsamen Absolvieren von Gruppenherausforderungen verabreden und so Punkte sammeln. Wettkämpfe in Aktionswochen und Highscores sowie die Möglichkeit, Erfolge in sozialen Netzwerken zu teilen, motivieren zusätzlich zu bewussten Mobilitätsentscheidungen.

Um den Fahrradverkehr in Enschede zu fördern, bekommen Fahrradfahrer bei Nutzung von SMART seit März 2018 Vorrang an einigen Ampeln. SMART erkennt Standort und Fortbewegungsmittel des Nutzers und kommuniziert mit dem Kontrollzentrum, um Grün anzufordern, wenn sich der Fahrer einer intelligenten Ampel nähert. Je besser die Anwendung die gewöhnlichen Routen eines Nutzers kennt, desto besser funktioniert der Service. Diese innovative Zusatzfunktion ist bei den Nutzern sehr beliebt und hat zu einem starken Zuwachs der Nutzungszahlen geführt.

Seit 2015 ist SMART Teil des EMPOWER Projektes der Europäischen Kommission. ${ }^{24}$ Ziel des Projektes ist die Förderung nachhaltiger Mobilität, um die Lebensqualität in Städten zu erhöhen. Mit Hilfe von innovativen Apps sollen die Bürger angeregt werden, seltener das eigene Auto und stattdessen umweltschonende Verkehrsmittel zu benutzen. Nachhaltige Mobilität soll belohnt werden. Im Rahmen des Projektes wurde SMART für die EMPOWER-Städte Milan (Italien), Göteborg (Schweden) und Newcastle (Vereinigtes Königreich) angepasst und als Forschungsprojekt implementiert, um weitere Erkenntnisse über Wirkungsweise von Anreizen für eine Änderung des Mobilitäts-

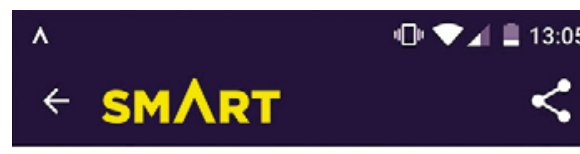

FIETS MET EEN GROEP MINIMAAL $1000 \mathrm{KM}$

Uitdaging loopt af op: 29 May 2016

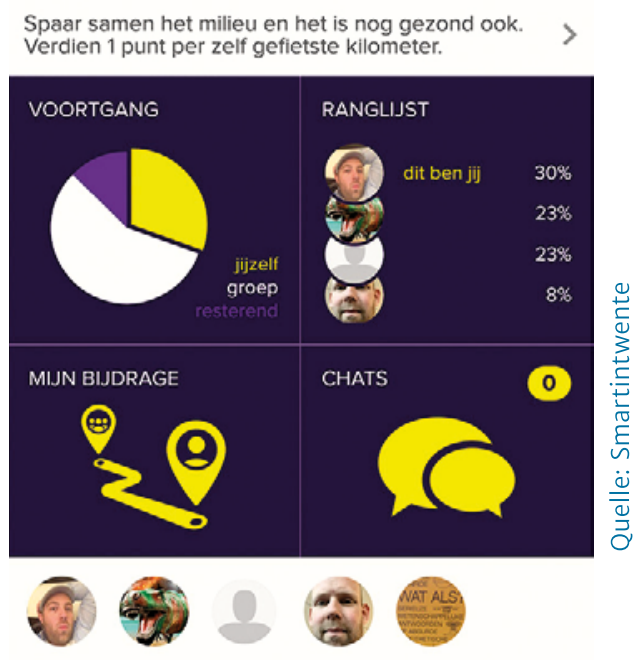

Abbildung 7 Nutzer können sich den Herausforderungen alleine stellen oder sich mit anderen Nutzern verabreden um Punkte zu sammeln verhaltens zu gewinnen. Im Anschluss an das Projekt nutzt nun auch Newcastle die App weiterhin für die Umsetzung der kommunalen Verkehrsstrategie. Eine ähnliche Anwendung von einem anderen Anbieter ist die App Liight, die 2018 in Madrid (Spanien) als Betaversion gestartet ist. Liight belohnt die durch Fahrradfahren und die Nutzung öffentlicher Verkehrsmittel eingesparte $\mathrm{CO} 2$ Emissionen mit Punkten, die für Rabattgutscheine und Produkte eingetauscht werden können.

\section{DATENBASIS UND -VERWENDUNG}

Nach Einverständnis der Nutzer läuft SMART auf dem Smartphone ständig im Hintergrund, um das individuelle Mobilitätsverhalten aufzuzeichnen. Mit Hilfe von GPS-Daten und Algorithmen erkennt SMART, welche Routen und welches Verkehrsmittel ein Nutzer wählt. Das erfolgreiche Absolvieren von Herausforderungen wird so automatisch erkannt und SMART Points werden gutgeschrieben. Historische Mobilitätsdaten der Nutzer werden in Benutzerprofilen zusammengefasst und dienen so einerseits für die Erstellung der individuellen Herausforderungen und liefern andererseits detaillierte 
Informationen für die kommunale Verkehrsplanung.

Zusätzlich liefert SMART auf Grundlage des Benutzerprofils und Standortes des Nutzers Informationen zur aktuellen Verkehrslage und zum Betriebsablauf der öffentlichen Verkehrsmitteln und schlägt, auch unter Einbeziehung von Wetterdaten, alternative Routen und Verkehrsmittel vor. Daten zur aktuellen Verkehrslage, wie Staus und Baustellen, werden von Google und der kommunalen Verkehrsüberwachung bezogen, Informationen zum Betriebsablauf der öffentlichen Verkehrsmittel werden über eine Schnittstelle des kommunalen Verkehrsunternehmens integriert. Wetterdaten bezieht SMART von einem öffentlichen Wetterdienst. In geringem Umfang werden auch Informationen zu aktuellen Vorkommnissen durch Nutzer eingetragen, die ebenfalls über die Anwendung veröffentlicht werden.

\section{FINANZIERUNG}

Die erste Entwicklung der App wurde durch die Gemeinde Enschede finanziert. Das EU-Förderprogramm Horizon2020 för-

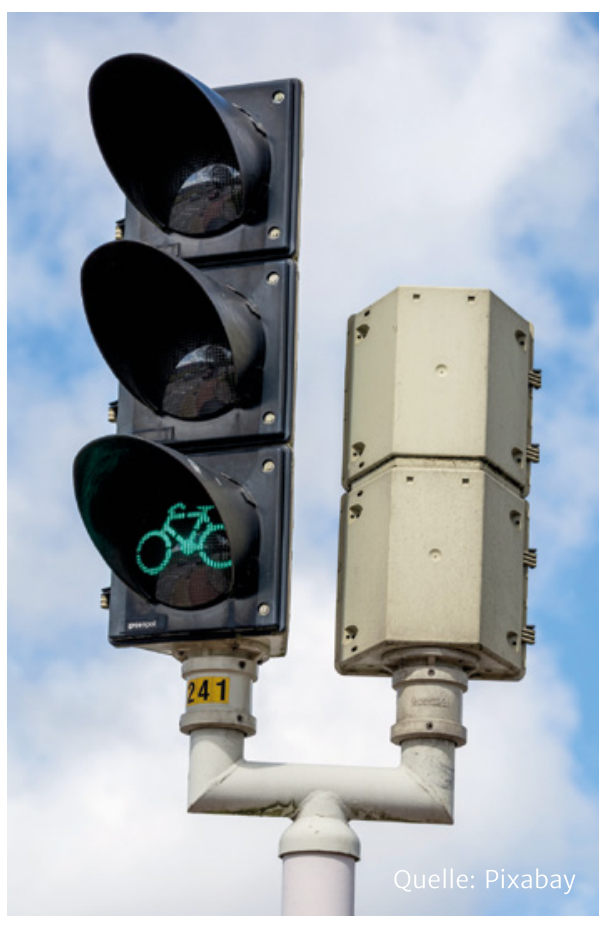

Abbildung 8: Fahrradfahrer, die SMART nutzen, bekommen an Ampeln Vorrang derte im Rahmen des Projektes SUNSET und des Nachfolgeprojektes EMPOWER die Weiterentwicklung der Anwendung und die Implementierung in weiteren Projektstädten. Der laufende Betrieb der Anwendung in Enschede erfordert ca. $100.000 €$ jährlich, die größtenteils von der Kommune getragen werden. Für die Nutzer ist die App kostenlos und werbefrei.

\section{ERFOLG}

SMART hat in Enschede etwa 2500 aktive Nutzer, die die App bei mindestens einer Fahrt verwendet haben. Verschiedene Aktionswochen und die neu eingeführte Bevorzugung an Ampeln haben zuletzt zu starken Nutzerzuwächsen geführt. Etwa 100 lokale Händler kooperieren mit SMART und stellen Gutscheine für die Anwendung zur Verfügung.

Die Stadt konnte mit Hilfe der App die Verkehrsströme so umlenken, dass Investitionen in Fahrbahnverbreiterungen sowie die Erweiterung eines Tunnels und einer Brücke eingespart werden konnten. Im Rahmen eines zweiwöchigen Aktionszeitraumes konnte zudem gemessen werden, dass mit Hilfe von SMART die mit dem Auto zurückgelegten Kilometer der App-Nutzer um bis zu 15\% gesenkt werden können. 


\section{PERSPEKTIVE}

Das Entwicklerteam plant, die Anwendung in Zukunft zu kommerzialisieren und in weiteren Städten, zunächst in den Niederlanden, einzuführen. SMART ist dabei vor allem für Kommunen interessant, die ein ganzheitliches Verkehrskonzept haben, da Kosten und Nutzen in verschiedenen Verwaltungsabteilungen anfallen können. Denkbar ist für die Entwickler auch eine Kommerzialisierung in Zusammenarbeit mit Unternehmen, die mit Hilfe der App umweltfreundliche Mobilität und Bewegung ihrer Beschäftigten belohnen wollen.

Die Gemeinde Enschede schätzt es, mit SMART ein Instrument zur Datenerhebung und zur Lenkung der Verkehrsströme in der Hand zu haben. Zudem erhält SMART zunehmend Bedeutung als Kommunikationskanal, mit dem die Stadt im Rahmen ihrer Strategie „Enschede Fietsstad 2020“ den Fahrradverkehr bewirbt. Mittelfristig wird allerdings angestrebt einen privaten Partner einzubinden, der den Betrieb der Anwendung übernehmen soll.

\section{Besonders interessant für Deutschland:}

- SMART trägt in enger Zusammenarbeit mit der kommunalen Verwaltung zur Umsetzung der kommunalen Verkehrsstrategie bei.

- Eine bessere Auslastung der Infrastruktur wird erreicht, indem die Wahl alternativer Routen belohnt wird.

- EU-Fördergelder stellen eine Möglichkeit für die gezielte Weiterentwicklung eines lokalen Projektes dar.

- Die Nutzerzahlen einer Anwendung können gesteigert werden indem für die Anwender ein unmittelbarer Vorteil entsteht (Grünphasen für Fahrradfahrer). 


\section{FALLBEISPIEL SAFEMOTOS}

\begin{tabular}{|c|c|c|}
\hline \multicolumn{3}{|l|}{ Kigali (Ruanda) } \\
\hline & $\begin{array}{l}\text { Anwendungs- } \\
\text { bereich }\end{array}$ & $\begin{array}{l}\text { Steigerung der Sicherheit von } \\
\text { Motorradtaxis für Kunden } \\
\text { und Fahrer }\end{array}$ \\
\hline & $\begin{array}{l}\text { Verwendete } \\
\text { Daten }\end{array}$ & $\begin{array}{l}\text { Mobilitätsdaten der Fahr- } \\
\text { zeuge, Bewertungen durch } \\
\text { Nutzer, (wenige) öffentlich } \\
\text { verfügbare Daten }\end{array}$ \\
\hline & Finanzierung & $\begin{array}{l}\text { Gebühr für getätigte Fahrten, } \\
\text { Teilfinanzierung durch GSMA } \\
\text { Fond }\end{array}$ \\
\hline & Zielgruppe & $\begin{array}{l}\text { Fahrer (gewerblich), private } \\
\text { und gewerbliche Nutzer von } \\
\text { Motorradtaxis }\end{array}$ \\
\hline
\end{tabular}

\section{BESCHREIBUNG DER ANWENDUNG}

SafeMotos ist eine Anwendung in Kigali, der Hauptstadt von Ruanda, welche die Nutzung von Motorradtaxis sicherer machen soll - sowohl für die Endkunden als auch für die Fahrer. Motorradtaxis gehören zu den am häufigsten benutzten Verkehrsmitteln in dem mittelafrikanischen Land, allerdings gibt es häufig Unfälle und Vorfälle von Gewalt gegen Fahrer und Endkunden. Hier setzt SafeMotos an, indem einerseits nur Fahrer eingesetzt werden, die bestimmte Sicherheitsstandards erfüllen. Andererseits sollen Übergriffe gegenüber den Fahrern und Endkunden dadurch reduziert werden, dass Profile mit persönlichen Daten der beiden Gruppen in der Anwendung sichtbar sind. Über eine App kann ein Kunde seit 2015 bei SafeMotos eine Fahrt bestellen. Die Anwendung wählt einen Fahrer in der Umgebung aus, der über einen bestimmten (von SafeMotos festgelegten) Sicherheitswert verfügt. Dieser Sicherheitswert wird auf Basis der bisher getätigten Fahrten ermittelt und stetig aktualisiert.

Die Fahrer erhalten hierbei ein Smartphone, auf der die SafeMotos App installiert wird. ${ }^{25}$ Mit Hilfe der App (oder einem On-Board Device mit speziellen Sensoren) können relevante Informationen zur Fahrweise erhoben werden, so dass in Echtzeit der Sicherheitswert einer Fahrt ausgewertet werden kann. SafeMotos setzt bewusst auf Fahrer mit mehrjähriger Erfahrung; neue Fahrer müssen erst 
einen bestimmten Sicherheitswert nachweisen, bevor sie als Fahrer bei SafeMotos aufgenommen werden können. ${ }^{26}$

Im Anschluss an die Fahrt ist ein Preis zu entrichten, der sich zunächst nach der Entfernung und der Fahrtdauer richtet. Zudem gibt es Aufschläge für Nachtfahrten, Wartezeiten bei den Fahrern und z. T. für gewerbliche Kunden (bspw. für geringe Wartezeiten der Kunden und weitere Auswahlfunktionalitäten). Ferner können weibliche Fahrgäste seit 2018 auch einen weiblichen Fahrer anfragen. Da derzeit noch sehr wenige weibliche Fahrerinnen für SafeMotos tätig sind, wird ein Aufschlag erhoben. Zukünftig sollen aber deutlich mehr weibliche Fahrerinnen ein-

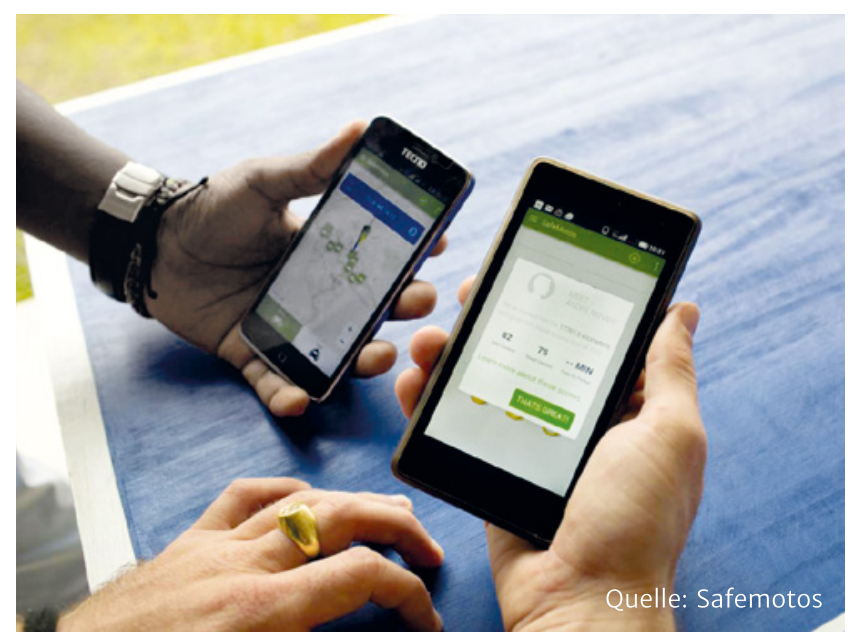

Abbildung 9: Fahrer bei SafeMotos müssen einen bestimmten Sicherheitswert nachweisen gesetzt werden, so dass dieser Aufschlag mittelfristig entfallen soll. Die Zahlung einer Fahrt erfolgt entweder durch Bar- oder Kartenzahlung oder über andere „cashless“ Apps. ${ }^{27}$

\section{DATENBASIS UND -VERWENDUNG}

SafeMotos greift auf verschiedene Datenquellen zurück: Primär werden Daten aus den Apps und On-Board Devices zur Fahrweise erhoben und ausgewertet, bspw. die Geschwindigkeit, Beschleunigungs- und Bremsverhalten sowie Erschütterungen bei der Fahrt. ${ }^{28}$ Hieraus wird durch Algorithmen ein Sicherheitswert für die einzelne Fahrt ermittelt. Zudem hat ein Kunde am Ende der Fahrt die Möglichkeit, den Fahrer zu bewerten. Auch eine Bewertung des Kunden durch den Fahrer ist im Rahmen der App möglich.

Weitere Feedbackfunktionalitäten - auch direktes Feedback zu SafeMotos - sind nutzbar. SafeMotos erwägt ferner, Daten für „Facial Recognition“ Funktionen zu verwenden. Dies soll die Gefahr von Übergriffen gegenüber Fahrern und Kunden weiter reduzieren.

Derzeit kann nur begrenzt auf externe Daten zurückgegriffen werden. Daten zu temporären Geschwindigkeitsbegrenzungen und zu Straßensperren sind häufig nur mit zeitlicher Verzögerung verfügbar, so dass die Qualität der Datenlage noch verbesserungswürdig ist. Die Gründer von SafeMotos streben an, künftig Wetterdaten in der App zu berücksichtigen. Da aufgrund der höheren Unfallgefahr die Nachfrage nach Fahrten bei Regen stark zurückgeht, soll die geospezifische Wetterlage in der App dargestellt werden. In diesem Fall können die Kunden die Begrenzung von Regengebieten erkennen und dann gegebenenfalls Fahrten zu alternativen Destinationen wählen, wenn der Regen bestimmte Straßen unbefahrbar macht. 


\section{FINANZIERUNG}

Die App finanziert sich über verschiedene Wege. Erstens ist für jede Fahrt ein Anteil am Entgelt (etwa 25\%) an SafeMotos zu entrichten. Die Höhe des Entgelts ist dabei immer auch so bemessen, dass sichergestellt ist, dass jede Fahrt für den Fahrer profitabel ist. Allein im letzten Jahr konnte dadurch der Umsatz um den Faktor 4 gesteigert werden.

Zweitens ist es SafeMotos gelungen, Investoren für das Projekt zu gewinnen und Anteile zu veräußern. Dies soll in Zukunft auch noch weiter fortgesetzt werden. Drittens erhält das Projekt

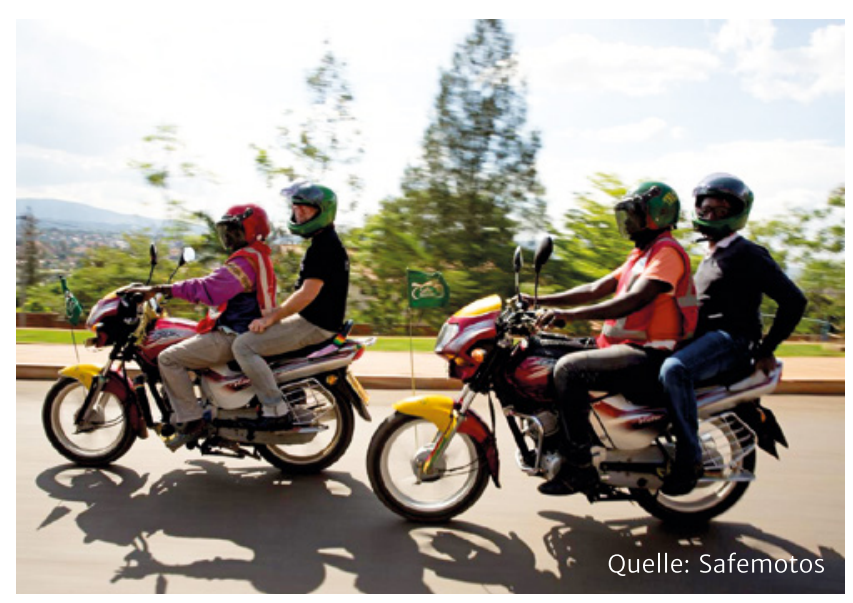

Abbildung 10. Die Sicherheitsstandards von SafeMotos erhöhen die Verkehrssicherheit in Ruanda finanzielle Förderung durch den GSMA (Global System for Mobile Communications) Innovation Fund, der Start-ups in Afrika und Asien unterstützt. ${ }^{29}$

\section{ERFOLG}

SafeMotos wurde mittlerweile bei ca. 2 Mio. Trips in Kigali und Umgebung eingesetzt. Hiervon wurden 500.000 durch die App auf Seiten der Endkunden initiiert. Die App wurde insgesamt etwa 58.000 Mal heruntergeladen; etwa 2000 User nutzen die App wöchentlich.

SafeMotos erhält sehr gute Bewertungen durch seine Kunden: Nach AIDS und anderen Krankheiten stellen Verkehrsunfälle aufgrund unsicherer Fahrzeuge und unsicherer Routen die häufigste Ursache für Todesfälle in Ruanda dar. In 80\% der Verkehrsunfälle in Ruanda sind Motorräder involviert. ${ }^{30}$ Auf dem Motoradtaximarkt in Ruanda gibt es aber sehr starke Unterschiede in Hinblick auf die Sicherheit. Aus Sicht der Kunden ermöglicht es die App, besser sichere Fahrer zu identifizieren.

Für die Fahrer stellt die Einstellung über SafeMotos eine Verbesserung der Arbeitsbedingungen sowie ein sicheres und stabiles Einkommen dar. Hierdurch wird aus ihrer Sicht ein wichtiger Schritt zur ökonomischer Selbstständigkeit getätigt.

Zum ersten Mal in der Geschichte Ruandas werden Frauen zu Motorradtaxifahrerinnen ausgebildet. Insbesondere Frauen als Kunden fragen gezielt weibliche Fahrerinnen nach, um die Gefahr eines Übergriffs zu reduzieren. Die Ausbildung von weiblichen Fahrerinnen erfolgt über das SafeMotos Institut in Kigali, einem US-basierten crowdfinanzierten Institut. ${ }^{31}$ Gerade für Frauen kann hierdurch ein erheblich sicheres Arbeitsumfeld geboten werden und eine wichtige Grundlage zur ökonomischen Selbstständigkeit und Empowerment gelegt werden.

Vgl. Announcing the first start-ups selected by our GSMA Ecosystem Accelerator Innovation Fund (2017), URL: www.gsma.com/ mobilefordevelopment/programme/ecosystem-accelerator/announcing-the-first-start-ups-selected-by-our-gsma-ecosystem-accelerator-innovation-fund/. 


\section{PERSPEKTIVE}

Perspektivisch soll SafeMotos auch in anderen Städten eingesetzt werden. SafeMotos möchte auf diese Weise das „Uber" für Motorradtaxis in der Region werden. Derzeit liegt der Fokus auf Ruanda, das einerseits aus Sicht der Gründer von SafeMotos gute Rahmenbedingungen für technologiebasierte Start-Ups bietet und andererseits über einen stark wachsenden IKT-Sektor verfügt, der auch durch staatliche Initiativen wie die Kigali Innovation City vorangetrieben wird. ${ }^{32}$

Die Gründer von SafeMotos prüfen derzeit mögliche Kooperationen mit anderen Branchen. Aus Sicht der Gründer sind etwa neue Geschäftsmodelle in den Bereichen Post und Logistik denkbar, die erst durch die Infrastruktur von SafeMotos entstehen können.

Denkbar ist aus Sicht der Gründer mittelfristig auch der Vertrieb von White Label Produkten (Geschäftssystem, Technologien, etc.) über Lizenzen in anderen Ländern. Die Umsetzung vor Ort müsste in diesen Fällen aber durch ein anderes Unternehmen erfolgen.

\section{Besonders interessant für Deutschland:}

- Einfache Bedienbarkeit und verschiedene Zahlungsmethoden können die Nutzerzahlen einer Anwendung steigern.

- Die Attraktivität der App wird aus Nutzersicht dadurch erhöht, dass finanzielle Anreize mit einer Erhöhung der Sicherheit verknüpft werden.

- Indem die Anwendung mögliche Gefahrenstellen zeitnah identifiziert und weitergibt, kann die Verkehrssicherheit deutlich erhöht und Stauungen vermieden werden.

- Durch Aus- und Weiterbildung der Fahrer können langfristige und verlässliche Arbeitsverhältnisse geschaffen werden. 


\section{FALLBEISPIEL URBANCYCLERS}

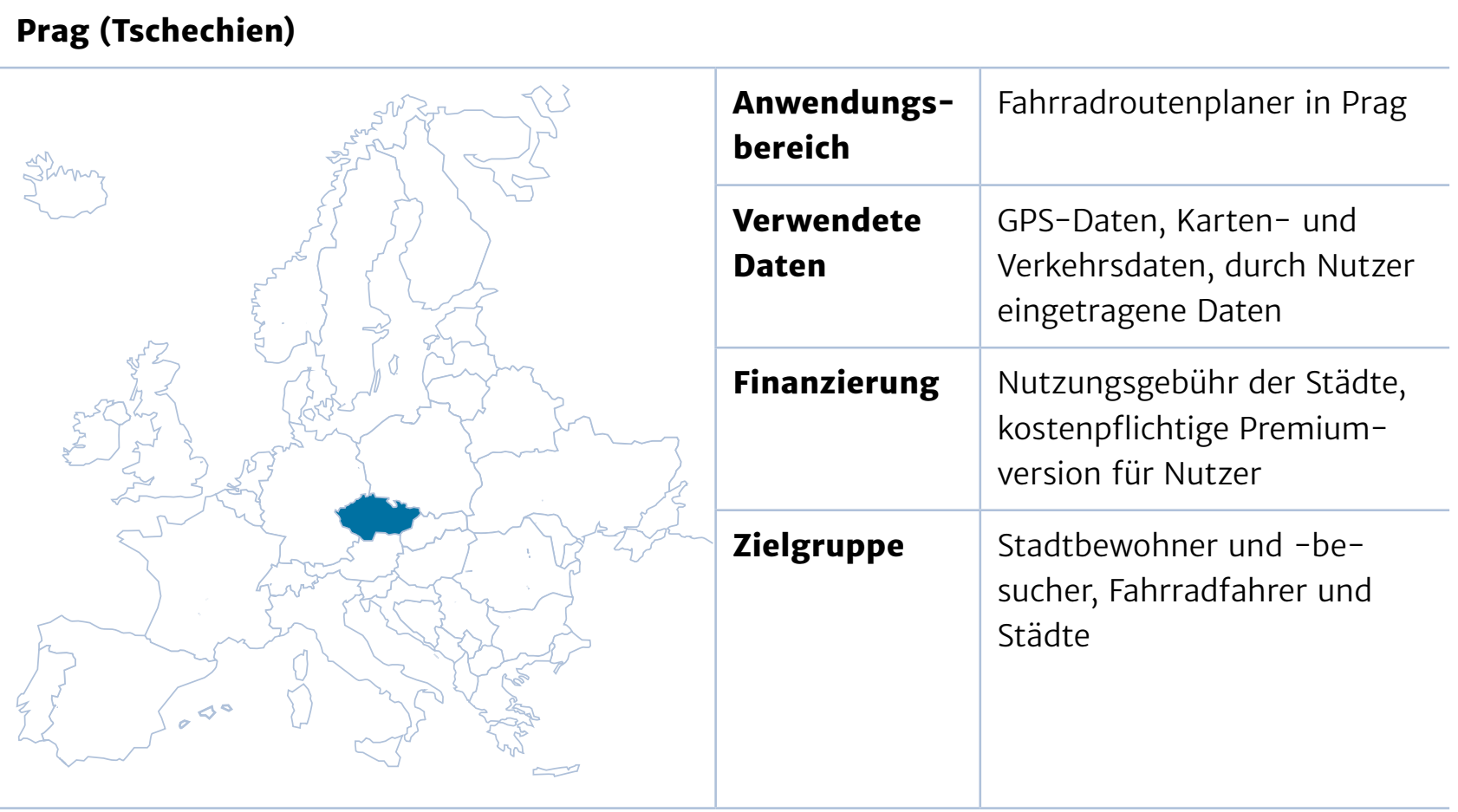

\section{BESCHREIBUNG DER ANWENDUNG}

UrbanCyclers ist ein intelligenter Fahrradroutenplaner. Viele der am Markt befindlichen Anwendungen nutzen für die Fahrradroutenberechnung Algorithmen, die ursprünglich für die Autonavigation entwickelt wurden. Die so generierten Routenempfehlungen sind daher oft unzureichend auf die Bedürfnisse der Fahrradfahrer abgestimmt, da sie neben der benötigten Zeit und Distanz nur begrenzt weitere Kriterien für die Wegfindung berücksichtigen. UrbanCyclers verknüpft Karten- und Verkehrsdaten unterschiedlicher Quellen mit eigenen Nutzerdaten, um sie mit Hilfe von Algorithmen auszuwerten. Dabei lernt die Anwendung kontinuierlich aus der Auswertung der Daten, um bestmöglich die von den Fahrradfahrern bevorzugten Routen vorzuschlagen.

Forschungsergebnissen über Vorlieben von Fahrradfahrern sowie häufige Unfallquellen werden zusammen mit Unfallstatistiken sowie den aufgezeichneten GPS-Daten der Nutzer analysiert, um Routenalternativen zu bewerten. Routenvorschläge lassen sich personalisieren, indem Nutzer angeben, welchen Fahrradtyp sie fahren (Citybike, Mountainbike, Rennrad, etc.) und welche Strecken sie bevorzugen (z. B. flache, wenig befahrene Straßen ohne Hindernisse). Darauf basierend schlägt UrbanCycler eine schnelle Strecke und eine besonders fahrradfreundliche Strecke als Routenalternativen vor. Die angegebene Fahrtdauer wird aus den aufgezeichneten Routendaten errechnet. Von anderen Nutzern eingetragene Gefahrenstellen oder Hinweise werden für die gewählte Route ebenfalls angezeigt.

Ein wichtiger Bestandteil der Routenplanung von UrbanCyclers ist Intermodalität. Je nach lokalen Kooperationspartnern kann die Fahrradroutenplanung mit öffentlichen Verkehrsmitteln und Bi- 


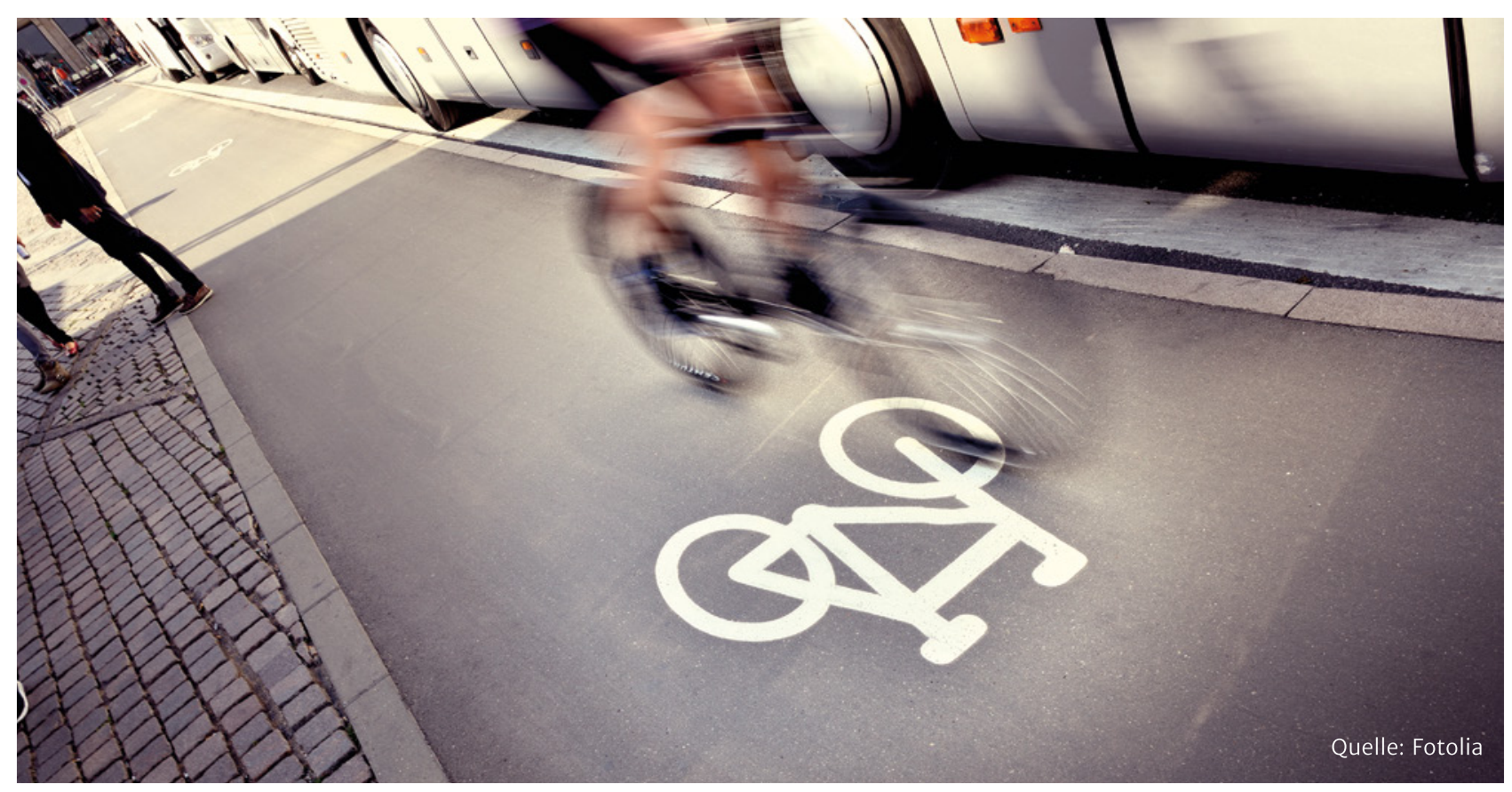

Abbildung 11: UrbanCyclers will die Fahrradnutzung in der Stadt fördern

ke-Sharing Angeboten verbunden werden. Standorte und Verfügbarkeiten der jeweiligen Anbieter sind in Echtzeit einsehbar.

Um Fahrradfahren und Nutzung der App zusätzlich zu motivieren, sind in der Anwendung spieltypische Elemente eingebunden („Gamifizierung(). Nutzer können sich virtuelle Auszeichnungen erfahren, reelle Preise gewinnen und sich mit anderen Nutzern in Wettbewerben messen. Belohnt wird u.a. häufiges aufzeichnen der gefahrenen Routen oder das Engagement der Nutzer, Hinweise und Empfehlungen zu Routen in der App einzutragen.

UrbanCyclers bietet außerdem generierte Daten und Verkehrsanalysen für Städte an, die so die Erkenntnisse des Routenplaners für ihre Verkehrsplanung nutzen können. Prag wertete mit Hilfe von UrbanCyclers aus, welche Einbahnstraßen für den Fahrradverkehr in beide Richtungen geöffnet werden können. Bislang konnten im Rahmen dieses Projektes etwa 70 Straßen freigegeben werden. Auch durch die Nutzer eingetragenen Hinweise zu Straßenschäden oder Hindernissen auf den Strecken können für die Städte von Interesse sein und sollen in Zukunft verfügbar gemacht werden.

UrbanCyclers wurde 2015 von einem Spin-Off von Studierenden und Beschäftigten der Technischen Universität Prag gegründet. Das Entwicklerteam will mit Hilfe der App die Fahrradnutzung in der Stadt fördern, indem sie eine bestmögliche Fahrradnavigation bereitstellt, spielerische Anreize zum Fahrradfahren bietet und Städte dabei unterstützt, ihre Infrastruktur für den Fahrradverkehr zu verbessern. Nach einer zweijährigen Entwicklungszeit ist die Anwendung seit 2017 als App und Webanwendung verfügbar. Die vollumfängliche Nutzung der App ist in Prag möglich, für weitere Städte weltweit sind Betaversionen verfügbar. Funktionen der sozialen Vernetzung sowie der Gamifizierung sind generell überall verfügbar. 


\section{DATENBASIS UND -VERWENDUNG}

Das Kartenmaterial von UrbanCyclers basiert auf OpenStreetMap, einer frei nutzbaren Geodatenbank. Von hier bezieht die Anwendung auch Daten zu Fahrradwegen, Fahrradabstellflächen, Straßenoberflächen, etc. Partnerstädte bzw. kommunale Verkehrsbetriebe stellen Fahrplandaten und Verkehrsdaten bereit.

Die zurückgelegten Routen der App-Anwender werden aufgezeichnet. Nutzer können gefahrene Strecken bewerten, eigene Empfehlungen abgeben und Kommentare zu möglichen Gefahrenstellen in der App eintragen. Diese Informationen können wiederum durch die Nutzer bewertet werden, um Richtigkeit und Relevanz zu gewährleisten.

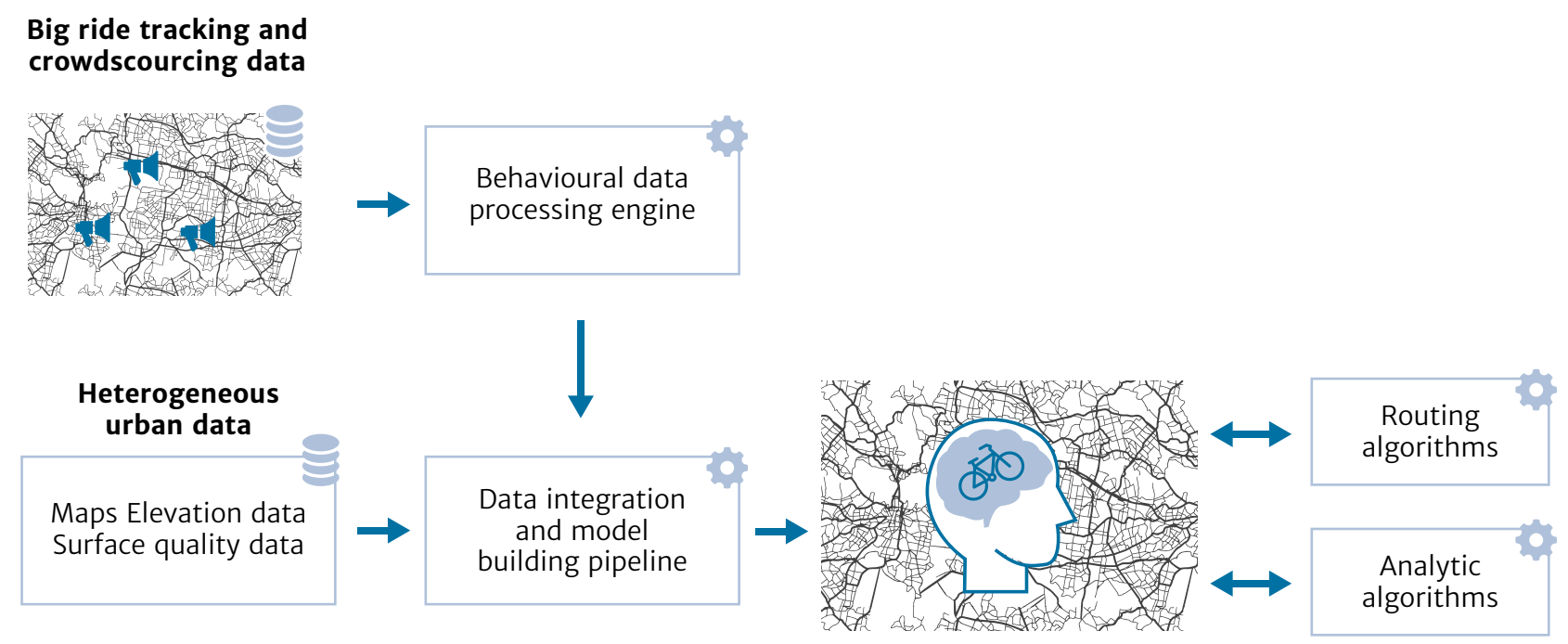

Abbildung 12: UrbanCyclers verknüpft von Nutzern erhobene Daten mit Karten- und Verkehrsdaten unterschiedlicher Quellen, um sie mit Algorithmen auszuwerten

\section{FINANZIERUNG}

UrbanCyclers finanziert sich hauptsächlich aus der Zusammenarbeit mit Städten. Die Anwendung wird als Software as a Service (SaaS) angeboten und kann als White-Label Produkt für jede Stadt angepasst werden. UrbanCyclers bildet bspw. die Grundlage der Smartphone-Anwendung „Prague on Bike," der offiziellen Fahrrad-App der Stadt Prag.

Wird die App nicht in Partnerschaft mit einer Stadt angeboten, ist die Nutzung generell in einer Basisversion kostenlos. Eine Premiumversion von UrbanCyclers, die erweiterte Einstellmöglichkeiten sowie eine Sprachausgabe bietet, ist gegen Zahlung erhältlich. Für die Zukunft ist geplant, Werbung und gesponserte Inhalte in die App aufzunehmen und so weitere Finanzierungsquellen zu erschließen. 


\section{ERFOLG}

Im Jahr 2017 wurde die App mehr als 50.000 Mal heruntergeladen, bei einer durchschnittlichen Bewertung von 4,5/5 Sternen im Google Playstore. Mehr als 250.000 Fahrten wurden mit der App bereits aufgenommen, das entspricht einer Strecke von ca. 3 Millionen zurückgelegten Kilometern. Die Auswertung einer von UrbanCyclers durchgeführten Umfrage ergab, dass 51\% der 460 befragten App-Nutzer öfter in der Stadt das Fahrrad benutzen, seitdem sie die App kennengelernt haben. Für 2018 ist die Durchführung einer Studie in Zusammenarbeit mit der Technischen Universität Prag geplant, die weitere Erkenntnisse über den Einfluss von UrbanCyclers auf das Mobilitätsverhalten untersuchen soll.

\section{PERSPEKTIVE}

UrbanCyclers plant, in weitere Städte weltweit zu expandieren, zunächst in Tschechien und Europa. Bereits jetzt sind Betaversionen für Berlin, London (Großbritannien), Mailand (Italien), Santiago de Chile (Chile) und Bogota (Kolumbien) verfügbar. Die spielerischen Elemente, mit denen UrbanCyclers das Fahrradfahren belohnt, lassen sich perspektivisch auch für die Förderung jeglicher aktiven oder nachhaltigen Mobilität nutzen. Denkbar ist eine Anpassung des Belohnungssystems an die Präferenzen der jeweiligen Stadt, sodass bspw. auch die Nutzung des ÖPNV belohnt werden kann.

\section{Besonders interessant für Deutschland:}

- Eine an die Bedürfnisse der Fahrradfahrer angepasste Routenplanung kann den Fahrradverkehr in Städten attraktiver und sicherer machen.

- Spielerische Elemente können die Benutzung alternativer Verkehrsmittel fördern.

- Die gesammelten und aufbereiteten Daten aus den Bewegungsprofilen der App-Nutzer können die Fahrradwegeplanung von Städten unterstützen.

- Eine weltweit verfügbare Basisversion der Anwendung ohne vollen Funktionsumfang kann eine Expansion in neue Märkte erleichtern. 


\section{SCHLUSSFOLGERUNGEN UND ERKENNTNISSE FÜR DEUTSCHLAND}

Innovative datenbasierte Anwendungen entstehen weltweit in den verschiedensten Sparten. Diese Studie stellt Anwendungsbeispiele aus den Bereichen Verkehrssicherheit, Hafenmanagement, Verkehrsplanung, intermodaler Routenplanung und nachhaltiger Mobilität vor. Innovative Aspekte entstehen hier durch die Anwendung neuer technologischer Lösungen auf bekannte Herausforderungen. Ihr Erfolg wird durch innovative Geschäftsmodelle, Finanzierungskonzepte und erfolgreiche Kooperationen zwischen verschiedenen Akteuren ermöglicht.

NordicWay ist ein länderübergreifendes Projekt zur Erhöhung der Verkehrssicherheit in Skandinavien, bei dem eine sichere und zuverlässige Kommunikation über kurze, mittlere und lange Entfernungen sichergestellt wird. Das Projekt macht den Nutzen internationaler Zusammenarbeit für internationale und örtliche Verkehre deutlich. Ein wesentlicher Erfolgsfaktor zur Steigerung der Verbreitung ist die Nutzbarkeit der Anwendungen über verschiedene Kanäle, etwa durch Smartphones und Bordcomputer im Auto der Endkunden.

Smart Port aus Estland ist ein Verkehrsmanagementsystem für den Fährverkehr im Hafen der Stadt Tallinn. Die Einführung eines neuen, zentralen Systems ermöglichte die Neugestaltung der gesamten Abfertigungsprozesse im Hafen, um die Wartezeiten für Autos und LKWs zu reduzieren und die Kapazitäten des Hafens zu erhöhen. Das Konzept für eine zentrale, automatisierte Verkehrssteuerung kann auch Anregungen für andere Fährhäfen, aber auch für das Parkflächenmanagement bei Großveranstaltungen liefern.

Trafi ermöglicht eine intermodale Routenplanung und den dazu passenden Ticketkauf aus einer Hand. Nutzer können so schnell und unkompliziert verschiedene Verkehrsmittel passgenau kombinieren, Mobilität wird vom individuellen Fahrzeugbesitz entkoppelt. Wesentlich für den Erfolg dieser Anwendung ist die Beteiligung der städtischen Mobilitätsanbieter, allen voran des ÖPNV. Mit Trafi kooperierende Städte können einerseits von einem attraktiveren Nahverkehr profitieren und andererseits gesammelte Daten für ihre Infrastrukturplanung nutzen.

Nutzer von SMART in Enschede (Niederlande) werden von der Anwendung mit Punkten belohnt, wenn sie mit umweltfreundlichen Verkehrsmitteln fahren oder bestimmte, weniger befahrene Routen wählen. Eine verlängerte Grünphase für Fahrradfahrer, die die App nutzen, hat darüber hinaus einen großen Nutzerzuwachs ermöglicht. Die Anwendung trägt in enger Zusammenarbeit mit der Stadtverwaltung von Enschede zur Umsetzung der kommunalen Verkehrsstrategie bei. EU-Förderprogramme ermöglichten die Weiterentwicklung der Anwendung und deren testweise Einführung in weiteren europäischen Städten.

SafeMotos ist eine Anwendung zur Erhöhung der Verkehrssicherheit von Motorradtaxis in Ruandas Hauptstadt Kigali. Sie bewertet Fahrer von Motorradtaxis auf Grundlage von Nutzerbewertungen sowie Bewegungs- und Erschütterungsdaten. Im Projekt wird auch eine gezielte Schulung und Weiterbildung der Fahrer vorgenommen. Nutzer und Fahrer profitieren gleichermaßen von der Erhöhung der Sicherheit und den finanziellen Anreizen im Rahmen der Anwendung. Die guten Nutzerzahlen 
resultieren auch dadurch, dass die Bedienbarkeit der Anwendung sehr einfach strukturiert ist und zwischen verschiedenen Zahlungsmöglichkeiten gewählt werden kann.

UrbanCyclers aus Prag ermöglicht eine an die Bedürfnisse von Fahrradfahrern angepasste Routenplanung. Indem die Anwendung die Fahrradnavigation mit spielerischen Elementen kombiniert, wird der Fahrradverkehr in der Stadt sicherer und attraktiver. Durch die Anwendung gewonnene und aufbereitete Daten können darüber hinaus von der Stadt für eine bedarfsgerechtere Infrastrukturplanung genutzt werden.

Die Fallstudien liefern Anregungen für datenbasierte Mobilitätsprojekte in Deutschland:

1. Mobilitätsanwendungen für Verkehrsteilnehmer sind dann erfolgreich, wenn sie einen echten Mehrwert für ihre Nutzer versprechen. Gute Beispiele sind Ampel-Grünphasen für Fahrradfahrer bei SMART (Enschede) und die integrierte Möglichkeit zur Bezahlung im intermodalen Routenplanungs-Tool Trafi

2. Anwendungen können erfolgreich sein, wenn sie spezifische Nutzerbedürfnisse berücksichtigen, zum Beispiel die Sicherheitsaspekte bei SafeMotos oder die spezifischen Bedürfnisse von Fahrradfahrern (Urban Cyclers), die von vielen multimodalen Routenplanern nur unzureichend adressiert werden.

3. Spielerische Elemente (Anreize der Gamifizierung) erhöhen die Akzeptanz und Motivation von Anwendungen für Privatkunden.

4. Kooperationen zwischen Kommunen und privaten Anbietern von Mobilitätsanwendungen können große Vorteile für beide Seiten generieren, siehe Urban Cyclers aus Prag oder SMART aus Enschede.

5. Bei der Verkehrsplanung in Häfen können große Effizienzgewinne erzielt werden, wenn logistische Prozesse digitalisiert und dabei komplett neu aufgesetzt werden, anstatt nur die vorhandenen Prozesse in die digitale Welt zu übertragen. 


\author{
Bildnachweise \\ istock // www.istock.de \\ Fotolia // www.fotolia.de \\ Pixabay // www.pixabay.com \\ Safemotos // www.safemotos.com \\ SMART // www.smartintwente.nl \\ Portoftallinn // www.portoftallinn.com \\ Trafi // www.trafi.com
}

Im Rahmen der Forschungsinitiative mFUND fördert das BMVI seit 2016 Forschungs- und Entwicklungsprojekte rund um digitale datenbasierte Anwendungen für die Mobilität 4.0. Mehr Informationen unter www.bmvi.de/mfund

Die mFUND-Begleitforschung des WIK unterstützt die effiziente und effektive Umsetzung des Förderprogramms. Mehr Informationen unter mfund.wik.org und @WIKnews 


\section{INNOVATIVE ANWENDUNGEN MIT MOBILITÄTSDATEN: INTERNATIONALE FALLBEISPIELE}

Eine Studie der mFUND-Begleitforschung des WIK

Autoren: Alex Dieke | Dr. Sebastian Tenbrock | Sonja Thiele |Julia Wielgosch | Oktober 2018 